\section{A) Check for updates}

Cite this: Nanoscale, 2020, 12, 15970

\title{
Recent progress in hybrid perovskite solar cells through scanning tunneling microscopy and spectroscopy
}

\author{
Sanjay Sahare, (D) a,b Prachi Ghoderao, ${ }^{\mathrm{c}}$ Sadaf Bashir Khan, (D) ${ }^{\mathrm{a}, \mathrm{b}}$ Yue Chan $^{\mathrm{a}}$ and \\ Shern-Long Lee (D) *a
}

\begin{abstract}
Currently, sustainable renewable energy sources are urgently required to fulfill the cumulative energy needs of the world's 7.8 billion population, since the conventional coal and fossil fuels will be exhausted soon. Photovoltaic devices are a direct and efficient means to produce a huge amount of energy to meet these energy targets. In particular, hybrid-perovskite-based photovoltaic devices merit special attention not only due to their exceptional efficiency for generating appreciable energy but also their tunable band gaps and the ease of device fabrication. However, the commercialization of such devices suffers from the instability of the compositional materials. The cause of instability is the perovskite's structure and its morphology at the sub-molecular level; thereby revealing and eliminating these instabilities are a striking challenge. To address this issue, scanning tunneling microscopy/spectroscopy (STM/STS) presents a comprehensive method to allow the visualization of the morphology and electronic structure of materials at atomic-level resolution. Here, we review the recent developments of perovskite-based solar cells (PSCs), the STM/STS analysis of photoactive halide/hybrid and oxide materials, and the real-time STM/STS investigation of electronic structures with defects and traps that are believed to mainly affect device performances. The detailed STM/STS analysis can facilitate a better understanding of the properties of materials at the nanoscale. This informative study may hold great promise to advance the development of stable PSCs under atmospheric conditions.
\end{abstract}

\begin{abstract}
Received 5th May 2020, Accepted 20th June 2020 DOI: $10.1039 /$ d0nr03499a rsc.li/nanoscale
\end{abstract}

\section{Introduction}

Energy consumption is becoming an international issue faced by everyone throughout the world. Sustainable and renewable energy resources are playing a crucial role in fulfilling the cumulative energy needs of the world's population. In this aspect, photovoltaic (PV) devices are a straightforward and highly efficient means to produce an enormous amount of energy. In particular, perovskite solar cells (PSCs) belong to an emerging thin-film PV cell class that consists of perovskite structured compounds and exhibits high energy conversion efficiency. Therefore, PSCs have become a global research hotspot in the PV arena. ${ }^{1-4}$ The energy conversion efficiency of PSCs has shown the steepest growth, which has crossed $24.5 \%$

\footnotetext{
${ }^{a}$ Institute for Advanced Study, Shenzhen University, Shenzhen, Guangdong, 518060 China.E-mail: sllee@szu.edu.cn

${ }^{b}$ Laboratory of Optoelectronic Devices and Systems of Ministry of Education and Guangdong Province, College of Optoelectronics Engineering, Shenzhen University, Shenzhen, Guangdong, 518060 China

${ }^{c}$ Department of Applied Physics, Defence Institute of Advanced Technology, Pune, 411025 India
}

thus far. ${ }^{5}$ Besides, it has been predicted that perovskites play a critical role in next-generation lasers, sensors, electric vehicle batteries, etc. ${ }^{6-8}$ However, the lack of a comprehensive understanding of the morphology and photo-physics of perovskitebased materials along with their stability issues largely hamper their progression..$^{9-11}$ The surface morphology and band energy levels directly influence the potential difference of a device, and thus the spatial transportation of charge carriers over time. ${ }^{12,13}$ Therefore, exploring the thin-film morphology and the electronic transport in perovskite materials is of key interest in this emergent field. The performance of perovskite-based optoelectronic devices is influenced by various electrical parameters, such as current-voltage $(I-V)$ hysteresis, trap states, charge carrier recombination, and ion migration, which are commonly defined by the morphologies of the film. ${ }^{14-18}$ Even a minute variation in sample preparation can lead to huge discrepancies in the resulting thin films. ${ }^{19-21}$ Consequently, changes in the aforementioned electrical parameters could determine the PV efficiency. Therefore, a thorough study of thin films at the nanoscale level is essential to reveal such structural problems to fabricate more efficient PV devices. ${ }^{22,23}$ 

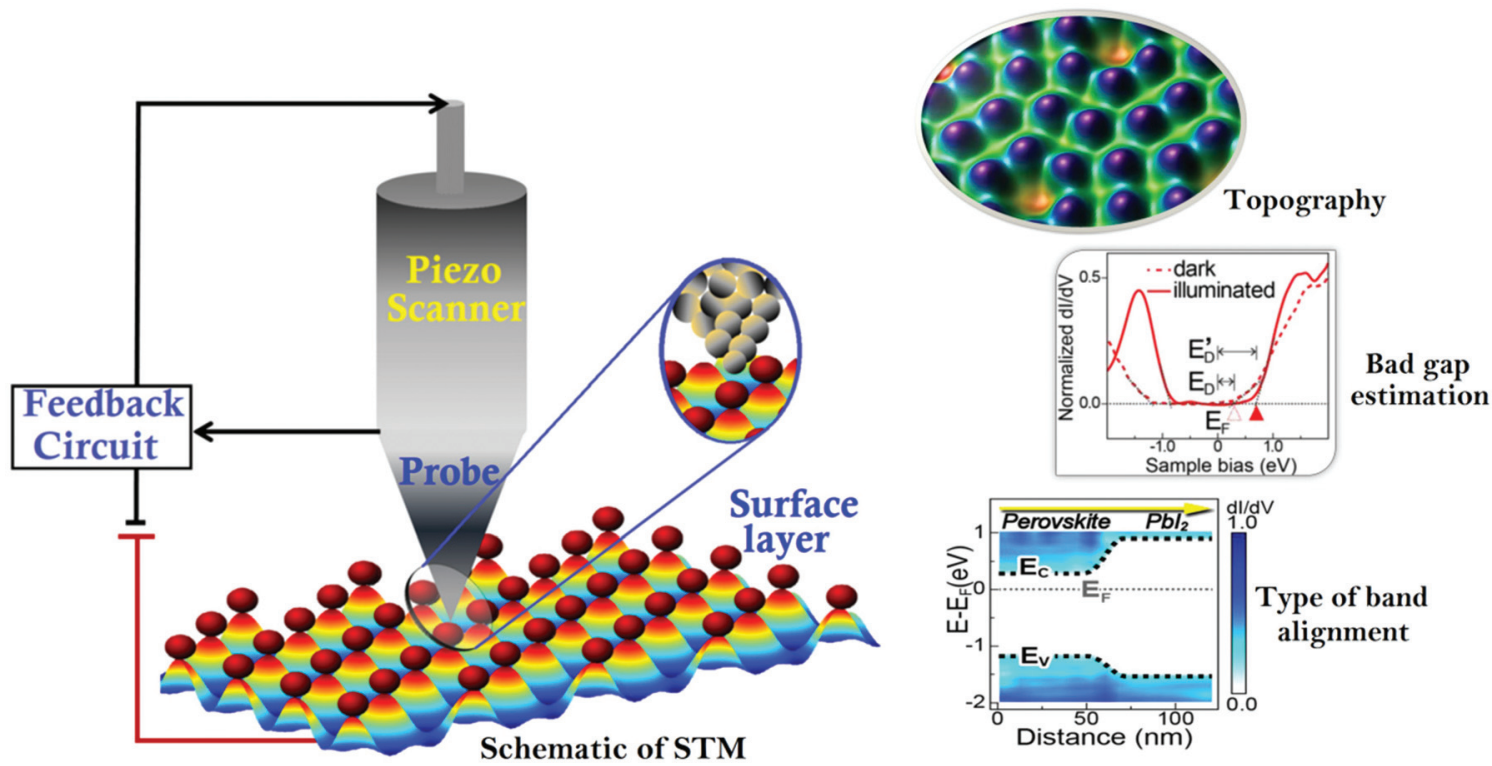

Fig. 1 An overview of the STM measurement technique and types of analyses (band gap estimation and alignment. Reproduced with permission from ref. 48).

Generally, X-ray diffraction (XRD), X-ray photoelectron spectroscopy (XPS), UV-Vis spectroscopy, photoluminescence, and $I-V$ measurements are common techniques for the detailed analysis of perovskite thin films. Unfortunately, these traditional techniques cannot provide real spatial resolutions. In this regard, scanning probe microscopy (SPM) has been utilized to obtain detailed information down to the atomic-level resolution of organic and inorganic PV materials. ${ }^{24-28}$ Numerous SPM techniques including scanning tunneling microscopy (STM), non-contact atomic force microscopy (AFM), Kelvin probe force microscopy (KPFM), and conductive-AFM (c-AFM) have been proposed to unveil the heterogeneities in perovskite thin films. ${ }^{29-34}$ Among AFM techniques, KPFM and c-AFM have been widely used to probe the photo-voltages and photo-currents of perovskite grains and their boundaries. In particular, KPFM, a non-contact AFM technique has contributed significantly to the identification of the local work function of samples by real-time visualization. ${ }^{30}$ This unique technique can provide surface information, including the composition and electronic states. Such a technique has been used to investigate the charge-separation locations, trap states, and different phases in perovskite materials by tracing the potential fluctuations. ${ }^{35-39}$ Similarly, STM can provide information about the structure, electronic properties, and electronic states (e.g., concerning the Fermi level of the electrode, Fig. 1) with a high spatial resolution $(0.1 \mathrm{~nm}) .{ }^{40,41}$ In contrast to c-AFM, the tunneling current between the STM tip and the sample surface is measured by moving the conductive tip without physical contact across the sample surface and thus no damage occurs to samples. According to the quantum mechanical phenom-

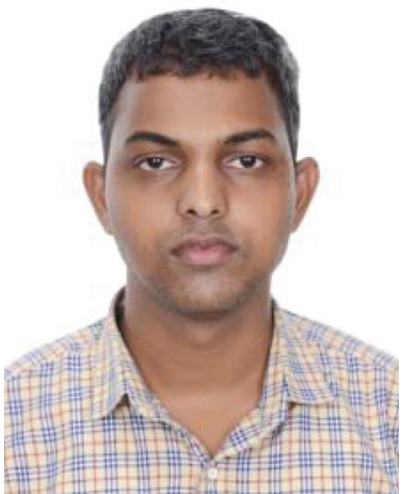

Sanjay Sahare
Sanjay Sahare is a Post-Doctoral fellow at Institute for Advanced Study, Shenzhen University, China. He received his Master's and Ph.D. from RTMNU, Nagpur, and Defence Institute of Advanced Technology, Pune, India, respectively. His research interests include Scanning probe microscopy, Micro fabrication techniques and Hybrid solar

cells.

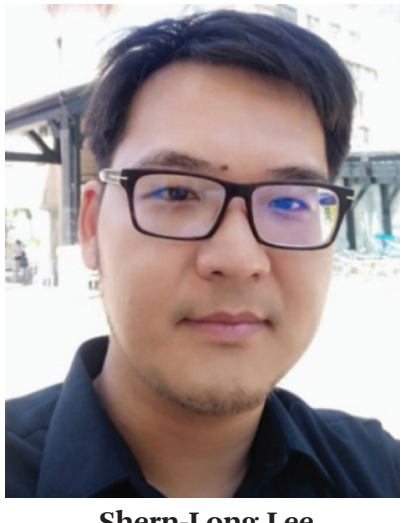

Shern-Long Lee received his PhD in chemistry in 2009 from National Taiwan University. During his doctoral work, he studied supramolecular selfassembly via scanning probe microscopy (advisor: Prof. Chunhsien Chen). In 2009-2012, he worked as a postdoc in the same group, after which he did his second postdoc (Marie Curie, 2012-2015) in Prof Steven De Feyter's group at $\mathrm{KU}$ Leuven in Belgium. He is now a researcher of IAS at Shenzhen University (China) where his research interests include materials and analytical chemistry. 
enon, the tunneling current between the STM tip and sample $(\sim 1.0 \mathrm{~nm})$ can occur once a voltage bias is applied. ${ }^{42}$ The tunneling current exponentially increases as the tip-sample distance reduces. This mechanism allows us to obtain high-resolution spatial images of surfaces of materials in real space (lateral resolution below $10 \mathrm{pm}$ and a vertical resolution $<1 \mathrm{pm}$ ). These defects at the atomic scale can strongly influence the behavior and performance of the photo-absorber layers and hence the efficiency of a device. One can see an overview of the STM measurement technique and its measurement ability (the type of sample analysis) at the atomic level in Fig. 1. This schematic depicts the formation of the tunneling junction between the sample and metallic tip, where electrons get tunneled from the sample to the tip or vice versa against the applied bias. ${ }^{43}$ Once the perfect topography is obtained at this particular location, the tunneling current can be measured. Furthermore, the measured current will be utilized to estimate the bandgap and density of states (DOS) of the materials. Also, the band alignment can be estimated for the appraisal of rapid charge transfer and its separation upon illumination at the heterojunction, which produces a photocurrent in the external circuit. ${ }^{44}$

To date, huge progress has been achieved in the performance enhancement of PSCs. Despite its significance, the analysis of PSCs via STM at the atomic scale has been rarely discussed. Abundantly existing literature reports have mentioned genuine hurdles regarding the issue of sample preparation; however the protocols to be followed for performing accurate STM measurements for PSCs remain elusive. ${ }^{45-47}$ So far, it remains a challenge to establish the specific sample-preparation methods for perovskite materials. Due to this limiting factor, the correlation of physical and chemical properties at the atomic level has not been thoroughly inspected in STM/STS analysis.

In this contribution, we present an overview of STM measurement techniques, where using a specific strategy, real-time electronic structures of the perovskite materials have been studied. Here, we discuss the STM analysis of two types of perovskite materials, namely, halide and oxide perovskite materials in the view of solar cell applications. In addition, we will discuss the sample preparation techniques/methods for STM investigations, which are crucial to obtain precise information regarding the interfacial properties of perovskite heterostructures. We anticipate that this review can offer substantial useful information, i.e. pros and cons of sample preparation that can extend toward atomic-level studies. These factors could be essential for researchers to assemble optimal materials in PV devices to enhance the stability and overall performance of hybrid PSCs.

\section{Perovskite synthesis and sample preparation techniques}

A specific sample-preparation procedure for better STM investigation is the most critical aspect to make a conclusive affirmation about the fundamental properties of perovskite materials. Technically, an immaculate and atomically flat surface is essential to obtain flawless STM images (morphology and structures), which are normally impossible to be obtained with perovskite materials. ${ }^{49}$ In this process, the substrate also plays an important role, since the morphology and topography of the film always depend upon the properties of the underlying substrate. ${ }^{50,51}$ Similarly, ITO/FTO coated glass, $\mathrm{Au}, \mathrm{Cr}$, and HOPG are commonly used as conducting substrates for electrical contacts in the process of STM sample preparation. ${ }^{52-54}$ Synthesis of a perovskite material is the first step towards the sample preparation for STM analysis. Researchers have used different methods to synthesize or prepare perovskite photon absorbing layers on different substrates. These synthesized samples can be used as it is, or sometimes we need to follow surface processing treatments (e.g. cutting and polishing) on it to make a more ideal sample for obtaining high-resolution STM images. ${ }^{47}$ The schematics of a few perovskite synthesis methods (e.g. solution process, vacuum, and chemical vapor deposition) and sample preparation techniques e.g. the cleaving method are shown in Fig. 2

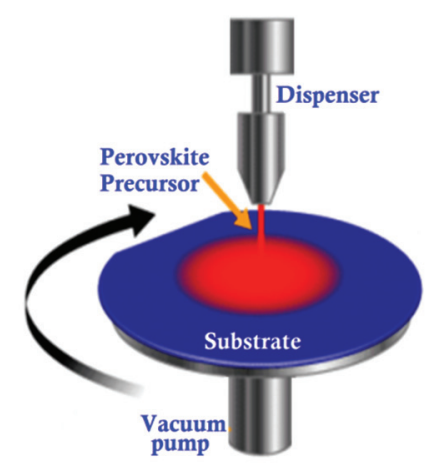

Solution Process Deposition

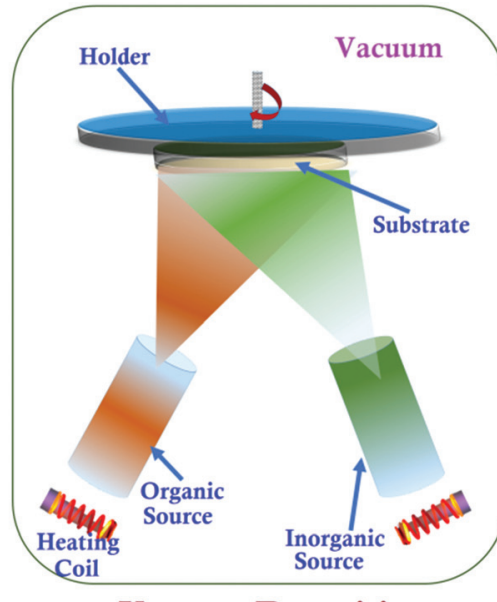

Vacuum Deposition

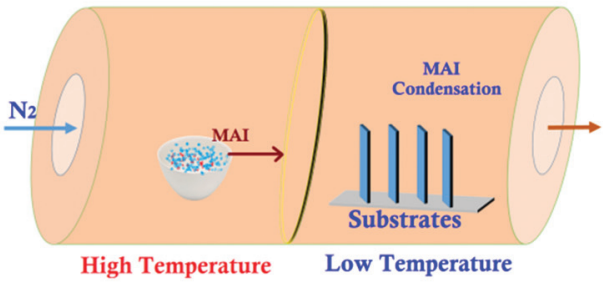

Chemical Vapour Deposition

Fig. 2 Perovskite synthesis/deposition methods. 


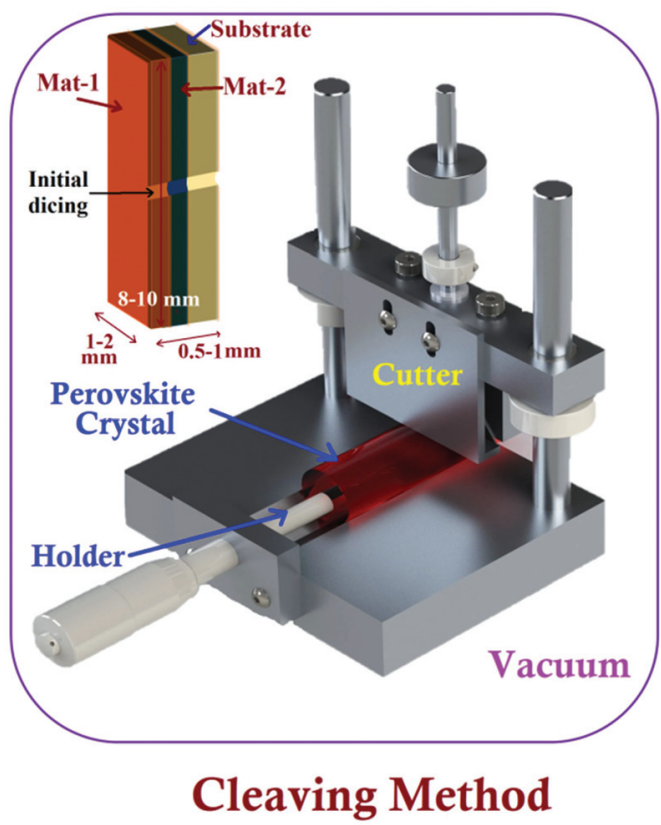

Fig. 3 Experimental set-up of the cleaving method to prepare XSTM/S samples. Inset figure shows basic sample preparation using initial dicing.

and 3, respectively. The details of these techniques are given as follows.

\subsection{Perovskite synthesis methods}

2.1.1. Solution process. Usually, perovskite thin films are prepared via the spin coating solution-process method owing to the ease of economically affordable processing. The potential of this technique for fabricating highly efficient photoactive layers has already been proven; however, the involvement of solvents and its exposure to air can contaminate the film surface. ${ }^{55}$ Moreover, the solution-processed technique has shortcomings such as lacking control over the crystallization process and the assurance of quality films. In short, this technique offers appraisal of both thermodynamic and kinetic effects to determine the morphology of the film. ${ }^{56}$ The inhomogeneity and multi-crystalline nature with a large rough surface can affect its sustainability and durability. L. Dou et al. have grown atomically flat and a few atomic unit thick 2D hybrid perovskites $\left[\left(\mathrm{C}_{4} \mathrm{H}_{9} \mathrm{NH}_{3}\right)_{2} \mathrm{PbBr}_{4}\right]$ directly on a substrate using the solution-process method. ${ }^{57}$ Azulay et al. have prepared single and mixed cation methylammonium (MA) lead iodide films in DMF, DMSO, and a combination of both solvents on $\mathrm{a} \mathrm{Au} / \mathrm{Cr}$ coated substrate. The solution concentration, spin rate, time, and temperature were optimized to obtain the desired thickness. ${ }^{58}$ Shih et al. prepared an $\mathrm{MAPbI}_{3}$ sample for light modulated STM measurements using a spin coating technique. ${ }^{48}$ The Si substrate was cleaned and processed with oxygen plasma before performing the sequential deposition of $\mathrm{MAPbI}_{3}$ on the dense $\mathrm{SiO}_{2}$ layer. Similarly, the precursor solution (1:3 ratio of $\mathrm{PbCl}_{2} / \mathrm{CH}_{3} \mathrm{NH}_{3} \mathrm{I}$ in DMF) was spin-coated onto an As-doped Si (100) substrate at $1500 \mathrm{rpm}$, followed by annealing $\left(90{ }^{\circ} \mathrm{C}\right.$ for $2 \mathrm{~h}$ ) under a nitrogen atmosphere. ${ }^{59}$ The deposited perovskite sample can be cleaved with the desired facet for carrying out an appropriate STM study.

2.1.2. Vacuum deposition. Another technique is known as vacuum deposition, which offers numerous advantages, including generating a smooth surface with high purity owing to the sublimation of precursor materials after extensive outgassing. ${ }^{60}$ Furthermore, these materials can be deposited on several substrates without any concern about solvent compatibility with other buffer layers (organic/inorganic films). ${ }^{61,62}$ Co-deposition has also been performed under ultrahigh vacuum $\left(1 \times 10^{-10} \mathrm{mbar}\right)$ for $\mathrm{MAPbI}_{3}$ film formation by L. She et al. $^{46,54}$ Single crystalline Au (111) was used as a substrate and cleaned by successive $\mathrm{Ar}^{+}$sputtering and annealing processes. $\mathrm{MAPbI}_{3}$ films have been grown on buffer layers by fixing a molar ratio of $1: 3$ for $\mathrm{CH}_{3} \mathrm{NH}_{3} \mathrm{I}$ and $\mathrm{PbI}_{2}$. The film was 10.8 atomic layer thick and smoother for further STM measurements. Likewise, an $\mathrm{MAPbBr}_{3}$ thin film was grown on a $\mathrm{Au}$ (111) single crystal surface by dual-source vacuum coevaporation of the MABr and $\mathrm{PbBr}_{2}$ species for 10 min at evaporation temperatures of 361 and $498 \mathrm{~K}$, respectively. ${ }^{63}$ On the other hand, in such a sequential co-evaporation technique, since the MAI layer was deposited over a metal halide layer, the MAI diffusion depth was limited, leaving the bottom metal halide layer unreacted. ${ }^{64}$ The stoichiometry of precursors (e.g., MAI and $\mathrm{PbI}_{2}$ ) has been controlled using a well-established and compatible technique for temperature-sensitive devices having high throughput and reliability in the electronic industries. The oxide perovskite (LSMO) has been deposited using pulse-laser deposition (PLD) on a Si substrate by Joshi et al. ${ }^{65}$ The growth conditions for the deposited films were optimized (KrF excimer laser, $248 \mathrm{~nm}$ wavelength, and $30 \mathrm{~ns}$ pulse width) and images were taken from the top surface of the deposited film. S. Wang et al. have examined the local electronic structure of oxide (perovskite) thin films deposited with a pulsed laser deposition system in conjunction with low-temperature STM measurements. In addition, they have modified the vacuum-deposition and developed a hybrid deposition technique by facilitating advanced tools and accessories to obtain an extremely smooth surface with high crystallinity, and the maximum surface coverage with a uniform chemical composition throughout the whole film. ${ }^{60}$

2.1.3. Chemical vapor deposition. The chemical vapor deposition (CVD) technique has many significant advantages, including precise control over the composition, highly crystalline nature, and the deposition on multiple and complex substrates, which also depend upon various deposition parameters (e.g., deposition time, gas flow rate, temperature, and pressure). ${ }^{66}$ These parameters are responsible for categorizing systems into sub-systems viz. Atmospheric Pressure and LowPressure CVD (APCVD and LPCVD), hot-wire CVD, hybrid physical CVD, metal-organic CVD, aerosol assisted CVD, etc., which have been used for the synthesis and fabrication of hybrid perovskite materials/films. ${ }^{67-69}$ For instance, Leyden et al. have initiated a vacuum/CVD deposition (hybrid) system to form perovskite layers. ${ }^{67}$ In brief, $\mathrm{PbCl}_{2}$ was initially de- 
posited using a vacuum deposition system, and subsequently loaded into a low-temperature zone. MAI, a cationic precursor, was allocated in the high-temperature zone to form a perovskite due to the condensation (in a low-temperature zone) under a uniform flow of $\mathrm{N}_{2} \cdot{ }^{70,71}$ These techniques, as mentioned earlier, have been used to prepare high quality and stable perovskites.

\subsection{Sample preparation techniques}

2.2.1. Cleaving method. The cleaving method is just another feasible method of sample preparation. However, obtaining a flat and clean surface by this method is quite difficult (unless the material is purely mono-crystalline). This technique has been widely utilized for III-V compound heterostructures and Si-based microelectronic devices at the nanoscale. ${ }^{72,73}$ Recently, Ohmann et al. have used an in situ (real-time) vacuum cleaving technique to prepare an STM sample from a hybrid perovskite $\left(\mathrm{MAPbBr}_{3}\right)$ for the first time. ${ }^{45}$ The cleaving of a single crystal in a specific orientation with the required flat surface is facile in the case of large-size crystals. Growing a large single-crystal, $\mathrm{MAPbBr}_{3}$, is easier than growing other halide perovskites due to its better stability in air. In other words, the success rate of obtaining a flat, immaculate surface from $\mathrm{MAPbBr}_{3}$ is slightly higher than those of $\mathrm{MAPbI}_{3}$ and $\mathrm{MAPbCl}_{3}$ owing to its larger crystal size. ${ }^{74} \mathrm{~A}$ schematic of the experimental set-up for sample preparation is shown in Fig. 3. Briefly, the sample was clamped as per the clamping style of the sample holder, and then cut half-way precisely with a dicing saw. Under UHV conditions, the sample was fractured by moving a rigid cleaver with a steady and slow force at the top portion of the sample above the cut. As soon as the applied force reaches its peak strength, a crack occurs in the cut region and propagates across the sample. The cleaving or fracturing process of the material depends upon the material's cleavage plane. ${ }^{75,76}$ The material having a cleavage plane is most eligible for the cleaving process, and becomes an ideal sample for STM measurements by creating an immaculate surface. On the other hand, the material without a cleavage plane may produce a bit rough surface owing to the fracturing process instead of cleaving. Mostly, in the $\mathrm{ABO}_{3}$ perovskite, the fracturing process occurs, and the surface is not ideal for STM measurements. ${ }^{77}$ Under such conditions, in situ annealing and etching processes may help achieve the goal of obtaining high-resolution images. ${ }^{49}$ Several researchers have also reported such a cleaving technique used for the STM analysis of non-PV single-crystal materials. ${ }^{78,79}$

The cross-sectional STM and STS (XSTM/S) are advanced techniques of STM, which can help achieve band mapping at heterojunction interfaces. ${ }^{80}$ This can be performed by creating a dirt-free surface of the cross-section by fracturing the material. ${ }^{76,81}$ For getting a sharp interface, the sample should be cut into long strips with the high precision dicing saw, where in situ fracture can be induced by applying steady force in a UHV environment. ${ }^{82}$ A schematic of the prepared strip is shown in the inset of Fig. 3. The success rate of XSTM/S sample preparation with an initial dicing process is about 70 to $80 \%$. For performing such experiments, special attention should be paid to the following factors such as obtaining a flat and defect-free cross-sectional surface to pin the Fermi level at the surface and, also, maintaining a specific height of the metallic tip consistently over the precise location of interest on the cross-sectional surface. ${ }^{83}$ Murali et al. have demonstrated their sample preparation methods under ultra-high vacuum (5 $\times 10^{-10}$ mbar) with a sharp blade, fixed on another sample holder, supported with a linear-rotary-motion feedthrough. ${ }^{84}$ The facets of the single crystal perovskite were sharply cut down into parallel planes with a size of $2-3 \mathrm{~mm}$, followed by in situ electron bombardment to achieve an atomic-scale surface reconstruction for STM imaging and measurements. In addition, hybrid perovskites were synthesized in the form of thin films using different methods. Therefore, these samples sometimes would not be suitable for interfacial/cross-sectional study due to their sizes, the underlying substrate, and other compatibility issues. In these circumstances, the samples need to be cleaved or fractured precisely in such a way that the desired facet can be viewed. Such an established system can be a potential platform for STM sample preparation and conducting studies to tune the surface properties at the atomic scale.

\section{Perovskite analysis by STM and STS}

STM and STS are the surface analytical techniques, capable of probing the DOS with a high spatial and energetic resolution. ${ }^{85,86}$ STM has contributed significantly to understand the sub-processes included in the fundamental phenomenon of PV. Notably, in organic solar cells, the exciton binding energy, electrostatic potential distribution, and charge transfer mechanism play an important role in intermolecular interactions of hybrid semiconductors. ${ }^{87,88}$ However, STM is a powerful technique to visualize the self-assembled monolayers at the atomic scale, from which one can correlate the performance of organic solar cells. ${ }^{89-91}$ When probing, a mechanically cut or chemically etched sharp tip is brought close to the sample surface $(\sim 2-10 \AA)$. A platinum-iridium (Pt-Ir) tip is commonly used to probe the sample due to its strength and stability. ${ }^{92,93}$ Electrons can tunnel into the empty states or accept it from the filled states depending on the applied bias (polarity) between the tip and the sample, deposited on the conductive substrate. This tunneling phenomenon occurs due to the energetic alignment between the work function of the tip and the sample energy levels with respect to the magnitude of the STM applied bias. Therefore, the level and location of conduction as well as the valence band (CB and VB) can be easily determined through the differential conductance $(\mathrm{d} I / \mathrm{d} V)$ spectrum (STS). The images obtained by STS can illustrate energy level mapping over the scanned area, particularly to analyze local electronic properties along with the visualization of a few hundred atoms accurately. ${ }^{44}$ In the view of significant challenges in obtaining local properties, here we will discuss 
the microscopic and spectroscopic analyses in halide/hybrid and oxide perovskites for PV applications.

\subsection{Halide perovskites}

As we discussed earlier, halide-perovskite-based solar cells are much more rapidly growing PVs. However, their commercialization is not yet possible because of their instability and toxicity. Various groups have analyzed thin-films and devices through different characterization techniques, and suggested fruitful solutions toward high-performance PVs, including the synthesis of defect-free perovskites, diverse deposition techniques, use of different transport layers and protective coatings, etc. ${ }^{94-99}$ The structural, morphological, and electrooptical characterization analyses provide an easy means to understand the behavior of perovskites, although as stated previously, atomic analysis is not yet fully explored due to sample constraints. Miyasaka et al. have used halide perovskites, e.g. $\mathrm{MAPbI}_{3}$, for the first time in dye-sensitized solar cells (DSSCs) as sensitizers in 2009. ${ }^{100}$ However, the sample preparation issues exist due to small crystal size and degradation, hence halide perovskite took a long duration to observe its atomic structures through STM. Until 2015, Ohmann et al. observed the atomic structure of $\mathrm{MAPbBr}_{3}$ with high spatial resolution by STM. ${ }^{45}$ These measurements were possible because of the bigger size single crystal that was easy to cleave and hence we can obtain a flat surface under ultra-high vacuum (UHV). One of the advantages of working under the UHV conditions is that one can obtain a superior control over the surface, which further makes it easier to study the single-molecule, submonolayer structures, and isolated clusters. ${ }^{101,102}$
3.1.1. Surface crystal structure and MA orientation. The atomic resolution measurement of $\mathrm{MAPbBr}_{3}$ through STM has helped significantly in understanding the ferroelectric and anti-ferroelectric features, ${ }^{45}$ which could be further responsible for the hysteresis effect in the halide perovskite solar cells. Fig. 4 shows the $\mathrm{MAPbBr}_{3}$ surface topographic image with an atomic resolution measured by in situ STM. One can observe bright spots (sphere) with a few angstrom corrugations that are arranged in an alternating manner with irregular distances between the next neighbors in both the directions (Fig. 4a, green line). In short, atoms were arranged in a zigzag fashion. Furthermore, systematic DFT calculations were performed using the VASP code to understand atoms and heterostructures of the specific molecules in detail. The orthorhombic crystal exists below the phase transition temperature $(T=144.5 \mathrm{~K})$. The calculated unit cell $(7.87 \AA \times 12.02 \AA \times$ $8.79 \AA)$ matches well with the experimental results of less than $3 \%$ deviation (Fig. 4b). The bright spot is identified as a $\mathrm{Br}$ anion, whereas the MA molecules are oriented perpendicular (or facing) to each other with an overall non-zero surface dipole that reflects a ferroelectric nature. Also, they observed that the surface MA groups are free to rotate in-plane and outof-plane after taking images from different domains. Similar coexisting structures are also observed, where the $\mathrm{Br}$ anion is paired and equally spaced dominating the earlier zigzag structure (Fig. 4c). Again, this structure was analyzed by calculating the surface with different in-plane orientations of MA. Interestingly, it shows the anti-ferroelectric alignment of MA cations (anti-parallel facing) due to the zero net dipole moment (Fig. 4d). Notably, each $\mathrm{Br}-\mathrm{Br}$ pair has two MA moi-
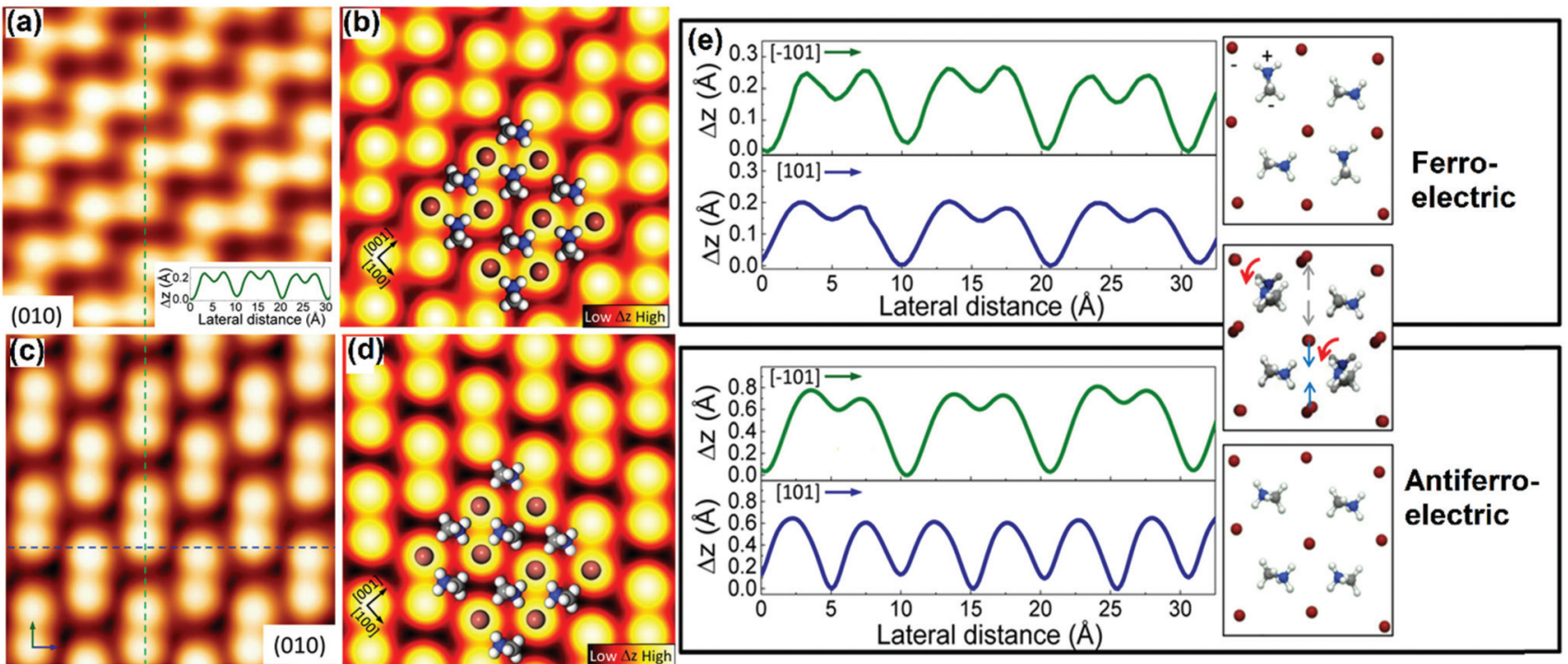

Fig. 4 (a) Atomic resolution of an $\mathrm{MAPbBr}_{3}$ resolved STM topography image of the perovskite surface. Height profile along the dashed line shown in the inset of (a); (b) simulated STM image of the (010) plane of the orthorhombic crystal with surface reconstruction. Br and MA ions are overlaid. The orientation of MA leads to different domains; (c) STM topographic image of another domain apart from that shown in (a). The arrows and dashed lines indicate the direction of the height profiles. (d) Corresponding calculated image of (c); (e) comparison of height profiles along perpendicular directions for two different observed domains and their corresponding models. Arrows indicate the rotational and positional changes of MA molecules and $\mathrm{Br}$ ions, respectively, between the ferroelectric and anti-ferroelectric domains. (a-e) Reproduced with permission from ref. 45. 
eties, which show dependency on the structure with different orientations related to each other. A significant structural difference has been observed by taking the height profile along two perpendicular directions as shown in Fig. 4e. In one direction (green line), one can see an alternating arrangement of short and long distances, whereas, in the horizontal direction, the atoms are equally spaced. These results clearly show the strong interplay between MA and $\mathrm{Br}$ ions that emphasizes the importance of the polar molecule present in the halide perovskite as a cation.

With regard to the electronic properties, DOS has been studied using STS, in which the tip gets stabilized at a fixed location above the surface with varying voltages. A typical STS spectrum (a derivative of $I-V$ ) and calculated DOS are shown in Fig. 5a and b. An interesting aspect arises, when only for a negative bias, it depicts a significant differential conductance $(\mathrm{d} I / \mathrm{d} V)$, and reveals the occupied states of the surface. On the other hand, it does not show an appreciable tunneling current indicating unoccupied states against the applied positive bias. This result is surprisingly consistent with STM images, and it is difficult to obtain topographic images upon an applied positive bias (not shown here). The partial DOS has been calculated and it strongly supports this observation (Fig. 5b). The DOS clearly illustrates the characteristics of ferroelectric and anti-ferroelectric domains leading to different electronic structures. Similarly, the dissimilar electronic phases tend to favor domain orientations in the normal direction of the thin-film surface. These orientations can alter the electronic properties of the materials.

The origin of the $\mathrm{MAPbI}_{3}$ perovskite surface and the phase transition between two surfaces have been revealed by She and co-workers. ${ }^{46}$ The zigzag and the dimer phase structures were found at MA-I-terminated (001) surfaces by STM. The zigzag row follows the original structure of the (001) planes in the bulk, and later on it exhibits iodine dimer rows with a shortened I-I distance for each dimer. The authors explained such iodine dimerization subsequently by net charge distribution and surface polarization. Fig. 6a shows an atomically smooth large-scale STM image of $\mathrm{MAPbI}_{3}$ films with step edges on the $\mathrm{Au}$ (111) substrate. High-resolution STM images showed two types of surface structures, namely the zigzag phase (Fig. 6b) and the dimer phase (Fig. 6c). The structures consist of a quasi-square unit cell with two bright protrusions per unit cell. In the zigzag phase, the bright protrusion (green rectangles) slightly deviated from the center, and redirected toward both the corners of the unit cell resulting in zigzag rows (Fig. 6b). The dimer phase formation was due to the bright protrusions shifting toward a corner of the unit cell (Fig. 6c). In addition, both phases were observed in the same region, with a height difference of less than 10 pm (Fig. 6d). The obtained orthorhombic perovskite structure with a periodic stacking of two $\mathrm{PbI}_{6}$ octahedra along the $c$-axis (001) is terminated by MA-I or Pb-I. The prediction of MA-I or Pb-I termination is made based on the induction of the tunneling current during STM scanning, where zigzag and dimer phases can convert into each other (Fig. $6 \mathrm{f}-\mathrm{i}$, ii, iii). In the process of phase conversion, in both cases, the band gaps of around 1.7 $\mathrm{eV}$ remain intact for the structures. The band gaps were measured at different locations of zigzag and dimer structures. MA-I or Pb-I termination has also been verified through DFT calculations. The simulated STM image is quite similar to that of the experimental result (Fig. 6b), in which the iodine anions denote bright dots and the MA cations are invisible in the figure confirming the MA-I-terminated surface of $\mathrm{MAPbI}_{3}$ films. DFT calculations supported the formation of I dimers, ascribed to the electrostatic interactions with the re-oriented MA cations. Similarly, $\mathrm{MAPbBr}_{3}$ surface reconstruction through Rashba splitting has been discussed. ${ }^{103}$ Special attention has been paid to the surface relaxation and the changes in their electronic properties. In the computational studies, the surface reconstruction of the (001) oriented facets of $\mathrm{MAPbBr}_{3}$ occurs in its orthorhombic phase, where the collec-
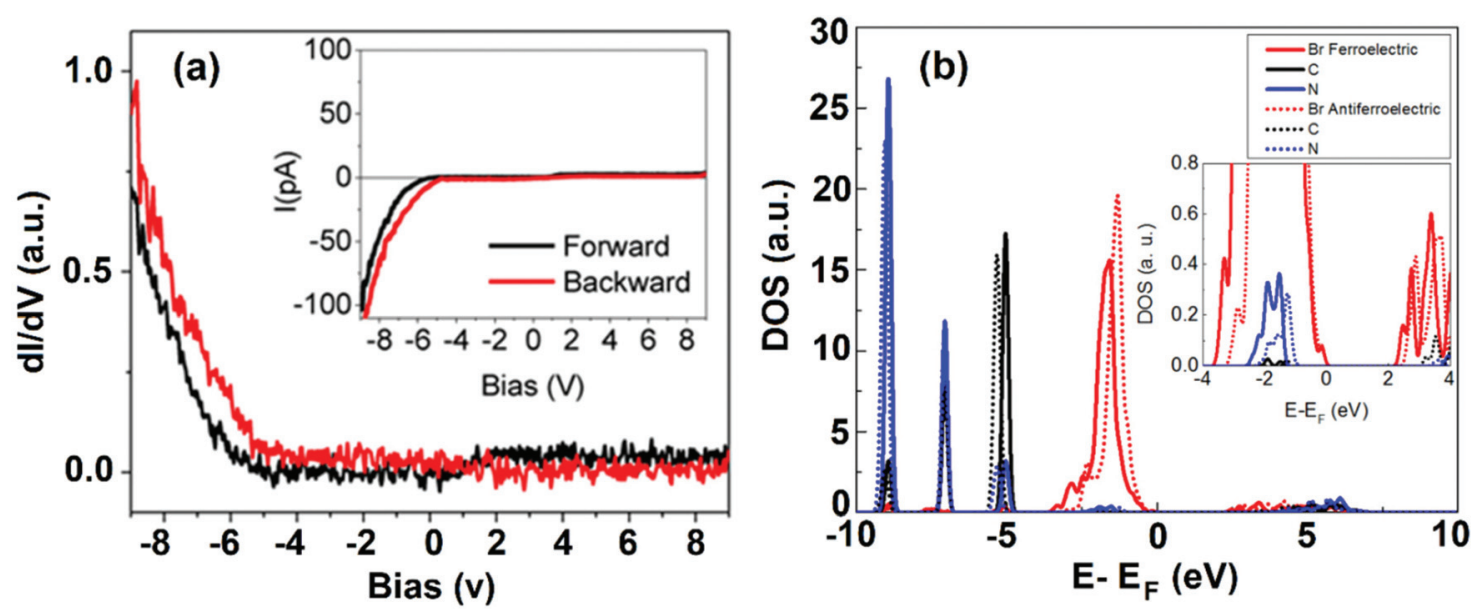

Fig. 5 (a) Scanning tunneling spectroscopy (d//dV) on the perovskite surface. Inset: Simultaneously acquired $I-V$ curve. (b) Comparison of density of states calculations on two different domains: ferroelectric (solid line) and anti-ferroelectric (dotted line). Inset shows a zoom-in of the energy region close to the Fermi level. (a,b) Reproduced with permission from ref. 45. 

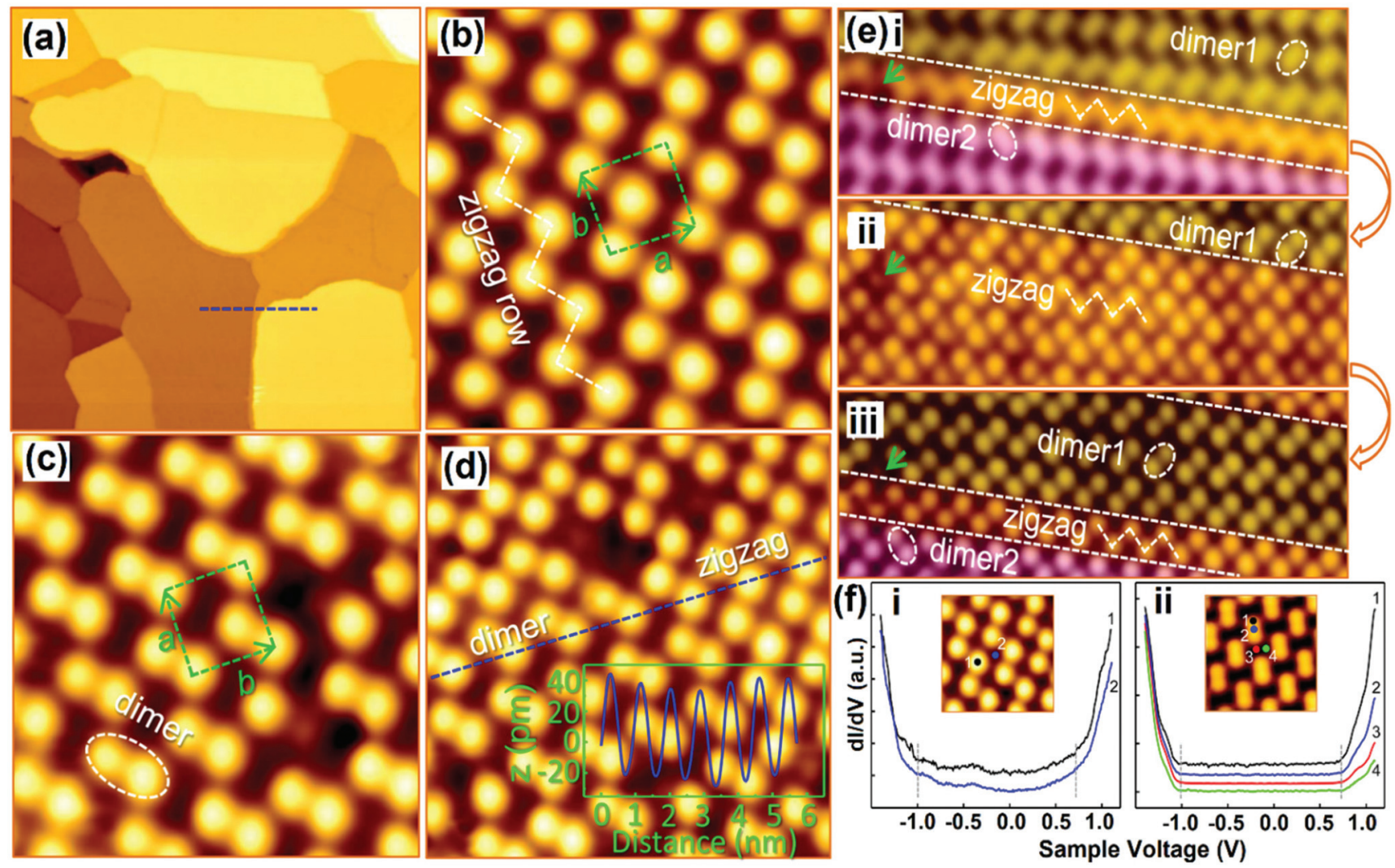

Fig. 6 STM images of MAPb 3 films deposited on Au(111). (a) Large-scale image $\left(300 \times 300 \mathrm{~nm}^{2}\right)$ with atomically flat terraces; (b and c) high-resolution images of the zigzag and dimer structures $\left(4.3 \times 4.3 \mathrm{~nm}^{2}\right)$. The unit cells are denoted by dashed rectangles. A zigzag row is indicated by the dashed line in (b), and an iodine dimer is denoted by a dashed ellipse in (c); (d) STM image of the two phases coexisting at the same region (5.6 $\times$ $\left.5.6 \mathrm{~nm}^{2}\right)$; (e-i, ii, iii) sequential STM images acquired at the same region showing the reversible transition between the dimer and zigzag structures $\left(4.2 \times 12.8 \mathrm{~nm}^{2}\right)$; and $(\mathrm{f}-\mathrm{i}, \mathrm{ii}) \mathrm{d} / \mathrm{d} V$ spectra acquired from different sites of the two surface structures: (i) zigzag and (ii) dimer. (a-f) Reproduced with permission from ref. 46.

tive orientations of MA cations generate the dimer and zigzag phases corroborating the structure provided by the previous STM experiments. ${ }^{46,63}$ Such analysis reveals the crucial role of MA cations as rotatable dipoles in the surface reconstruction and phase transition so that researchers can benefit from understanding the microscopic behavior of halide perovskites.

3.1.2. Defects. Different types of intrinsic defects viz. cationic, anionic, halide excess, and anti-site defects are responsible for the dynamic behavior of halide perovskites at the atomic scale. The variation in the PV efficiency of halide perovskites depends on the facet orientation of a crystal. Leblebici et al. have correlated the $\mathrm{PV}$ performance experimentally $\left(\mathrm{MAPbI}_{3-x} \mathrm{Cl}_{x}\right.$ based device) with the facet orientation using photoconductive AFM to explain the microscopic phenomenon. ${ }^{104}$

Furthermore, Stecker et al. have demonstrated the ion transport mechanisms at the surface, in the crystal direction, by both the theoretical and experimental approaches at the atomic level. ${ }^{63}$ They have further explored and confirmed the occurrence of vacancy-assisted transportation of the separate ions and vacancy defect clusters at the $\mathrm{MAPbBr}_{3}$ surface. In particular, the identification of intrinsic vacancies/defects on the surface of $\mathrm{MAPbBr}_{3}$ is shown in Fig. 7. The pristine surface ions of $\mathrm{MAPbBr}_{3}$ are also shown in Fig. 7a, where a bright protrusion depicts a $\mathrm{Br}^{-}$ion. The pairing took place due to the electrostatic interactions between two adjacent $\mathrm{Br}$ anions with positive nitrogen atoms (blue atoms, Fig. 7a), in which defect occurs. One can observe different types of intrinsic defects at the atomic scale from the STM image. An unpaired $\mathrm{Br}$ anion defect occurs because of the pair orientation mismatch in the same row (Fig. 7b). If some nearby vacancies occur, the adjacent unpaired Br defects can be observed (Fig. 7c). These vacancies are responsible for forming another type of defect, observed as dark depressions in the STM image (Fig. 7d). Subsequently, this leads to the formation of double and triple vacancies/defects (Fig. 7e and f). Theoretical calculations show that for a single $\mathrm{MABr}$ vacancy, there is a $90^{\circ}$ rotation of the MA cation close to the defect with the nitrogen end of the dipole near the unpaired $\mathrm{Br}^{-}$(Fig. $7 \mathrm{~g}$, dashed black circle).

The formation of double and triple MABr vacancies (Fig. $7 \mathrm{~h}$ and i) was confirmed by the theoretical energy values. At the perovskite surface, the Br-pair reorientation is the main cause of the dynamics. The (010) surface was reconstructed by orienting the Br-pairs along either the (101) or (10-1) direction, which are orthogonal to each other (green and purple rec- 


\section{Pristine Surface}

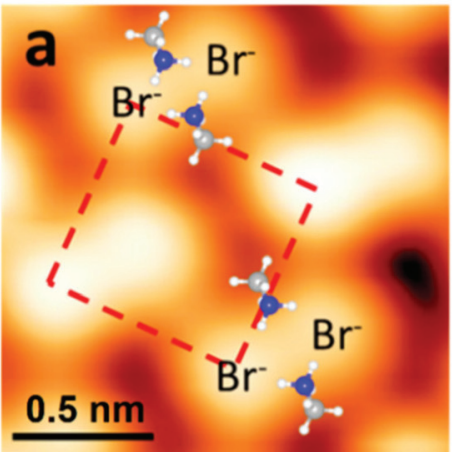

Single Vacancy

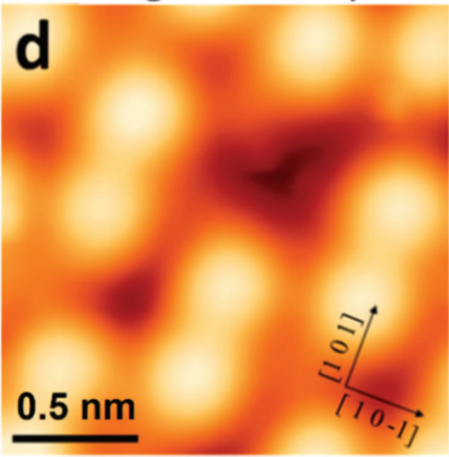

g

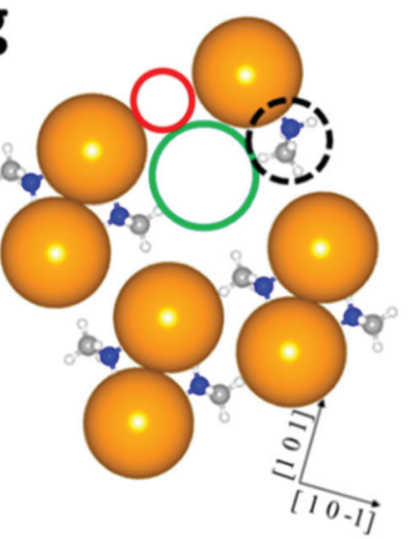

Unpaired $\mathrm{Br}$

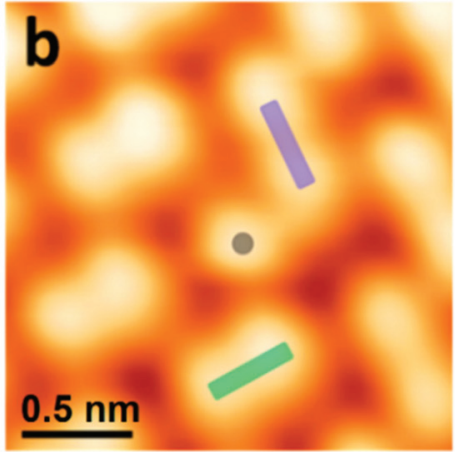

Double Vacancy

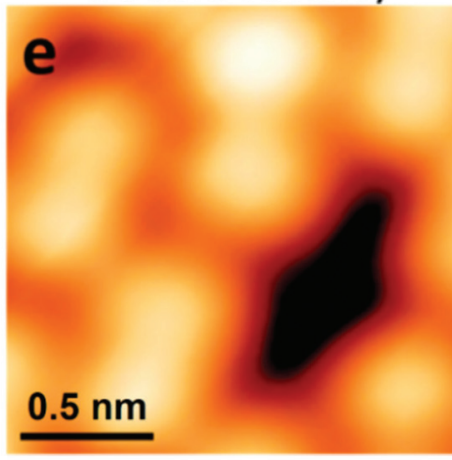

h

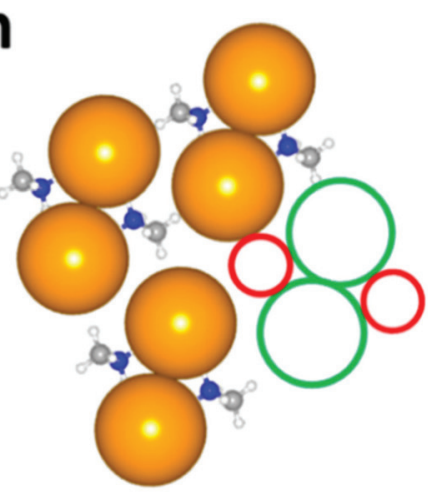

Two Unpaired $\mathrm{Br}$

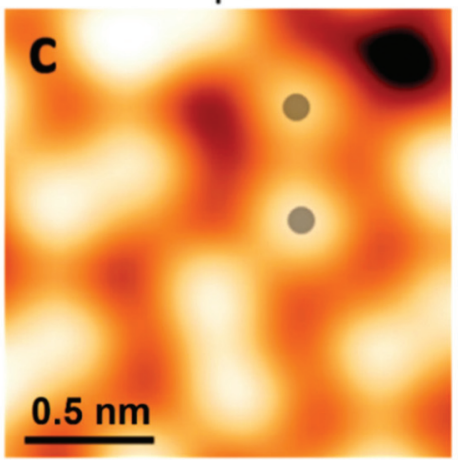

Triple Vacancy

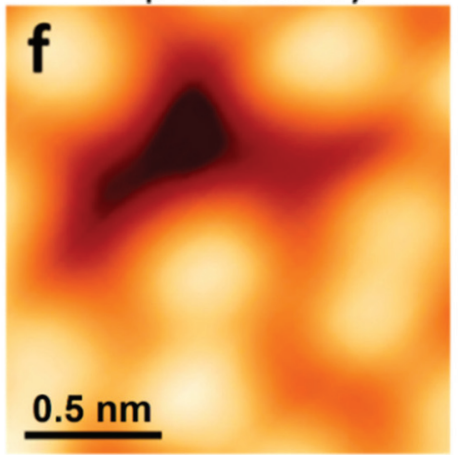

i

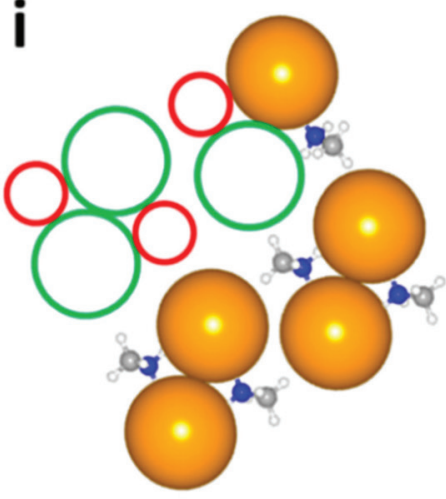

Fig. 7 Intrinsic defects at the atomic scale on the surface of $\mathrm{MAPbBr}_{3}$. (a) STM image of the pristine $\mathrm{MAPbBr}_{3}$ surface, with $\mathrm{MA}^{+}$molecules overlaid to show the relative position. The dashed red square denotes the unit cell of the $\mathrm{MAPbBr}_{3}$ (010) surface. (b) STM image of an unpaired Br (gray dot) at the point where two $\mathrm{Br}$-pair orientations (green and purple rectangles) meet. (c) STM image of two adjacent unpaired $\mathrm{Br}^{-}$(two gray dots), located near a vacancy. (d-f) STM images of single, double, and triple vacancy defects, respectively. ( $g-i)$ Top view of the model slab used for DFT defect formation energy calculations for the single, double, and triple vacancy defect cases, respectively. Solid green and red circles denote $\mathrm{Br}^{-}$and $\mathrm{MA}^{+}$ vacancy locations, respectively. The dashed black circle in (g) highlights a rotated MA ${ }^{+}$molecule. $(\mathrm{a}-\mathrm{g})$ Reproduced with permission from ref. 63.

tangles in Fig. 7b). Dissociation and re-association of the $\mathrm{Br}^{-}$ pair with the neighboring $\mathrm{Br}$ ions might be the reason for this reconstruction. The $\mathrm{MA}^{+}$rotation and $\mathrm{Br}^{-}$pair separation are also an important step for the occurrence of the $\mathrm{Br}^{-}$pair reorientation. This suggests that the reorientation event is more energetically favorable near a vacancy defect. An understanding of such defects at the perovskite surface or the heterojunction interface will enable direct utility in charge transport layers or electrodes of PV devices, since these have a direct impact on the charge carrier dynamics.
3.1.3. Influence of ambient exposure on the surface structure. The secret of PV performance reproducibility lies in the perovskite structure. The external environmental factors e.g. air and humidity may modify the structure, and are responsible for degradation of its optoelectronic properties. Several previous studies have revealed the degradation of $\mathrm{MAPbI}_{3}$ under humid air conditions by decomposition of $\mathrm{MAPbI}_{3}$ into $\mathrm{PbI}_{2}$ and MAI. ${ }^{105-109}$ In the degradation process, various mechanisms are involved including polymorphic transformation, hydration, decomposition, and oxidation. Similarly, the 
surface and structural modifications of $\mathrm{MAPbBr}_{3}$ have been investigated through XPS, PES, UV-Vis spectroscopy, etc. under ambient conditions. ${ }^{110-112}$ Murali and co-workers have demonstrated the surface structural transformation mechanism in $\mathrm{MAPbBr}_{3}$ using STM, where the single-crystal perovskite converts into a polycrystalline perovskite. ${ }^{84}$ They observed that this effect generally occurs under the ambient conditions, if the sample remains under those conditions for a longer duration. Specifically, their study involved two different samples: (i) a surface of an air-exposed as-grown single crystal, denoted as 'aged'; and (ii) a cleaved sample surface under UHV called 'pristine'. The topographic image of the aged surface divulges the prominent clusters and mounds throughout the surface (Fig. 8a). Such an effect is due to the hydrated perovskite crystal, which is swollen by the lattice expansion because of the local stress. ${ }^{113,114}$ This could be one of the reasons for the polycrystalline transformation from single-crystal $\mathrm{MAPbBr}_{3}$. A pristine surface that cleaved parallel to one facet of the crystal depicts the crystalline nature with layer-by-layer stacking as shown in Fig. 8b. There might be many substantial defects occurring on the pristine surface, which mainly depend on the perfection of crystal cleaving. The high-resolution topographic STM image (Fig. 8c) shows an ordered structure with consistent bright protuberances captured at a positive bias $(2.5 \mathrm{~V})$ that exhibits $\mathrm{Pb}$-rich octahedra with a $\mathrm{Pb}$ atom at the center. The ordered bright protrusions are distorted from an ideal crystal structure, ascribed to the lattice strain. This makes the octahe- dra tilt by changing an angle of the metal-halide-metal. Fig. $8 \mathrm{~d}$ illustrates the distances between two $\mathrm{Pb}$ atoms, where the step height of each layer is $1.48 \mathrm{~nm}$ and $0.52 \pm 0.02 \mathrm{~nm}$, respectively. To investigate the effect of ambient air on the pristine surface of the crystals, controlled experiments were also carried out for STM analysis. Upon overnight exposure to ambient air, the hydrated phases (hygroscopic nature) were observed leading to the degradation of halide perovskites. Fig. 8e and $\mathrm{f}$ depict the hydrate formation with ring-like protrusions (marked by circles) and the dis-unified 1D chains (marked by rectangles). The direct visualization of the surface transformation of singlecrystal halide perovskites via STM at atomic spatial resolution reveals the defects that are present in the crystal, consequently compromising the device performance.

3.1.4. Surface states and Fermi level pinning. Energy level modulation can be achieved by modifying organic cations in a halide or hybrid perovskite, which alters the performance of the devices. Zu et al. showed that donor-like surface states originating from reduced $\mathrm{Pb}$ instantaneously affect the band alignment at the perovskite/electron acceptor interface. ${ }^{115} \mathrm{Kim}$ et al. have examined mixed anion materials, which can be merged into the shallow levels in the CB to amend the charge kinetics. ${ }^{116}$ The direct evidence of such states has been revealed by Azulay et al. through STS, where they investigated the role of recombination centers within the bandgap. ${ }^{58}$ Modified bandgaps could directly alter the PV performance. In a $\mathrm{MAPbI}_{3}$ halide perovskite, they depict a Fermi energy $\left(E_{\mathrm{F}}\right)$
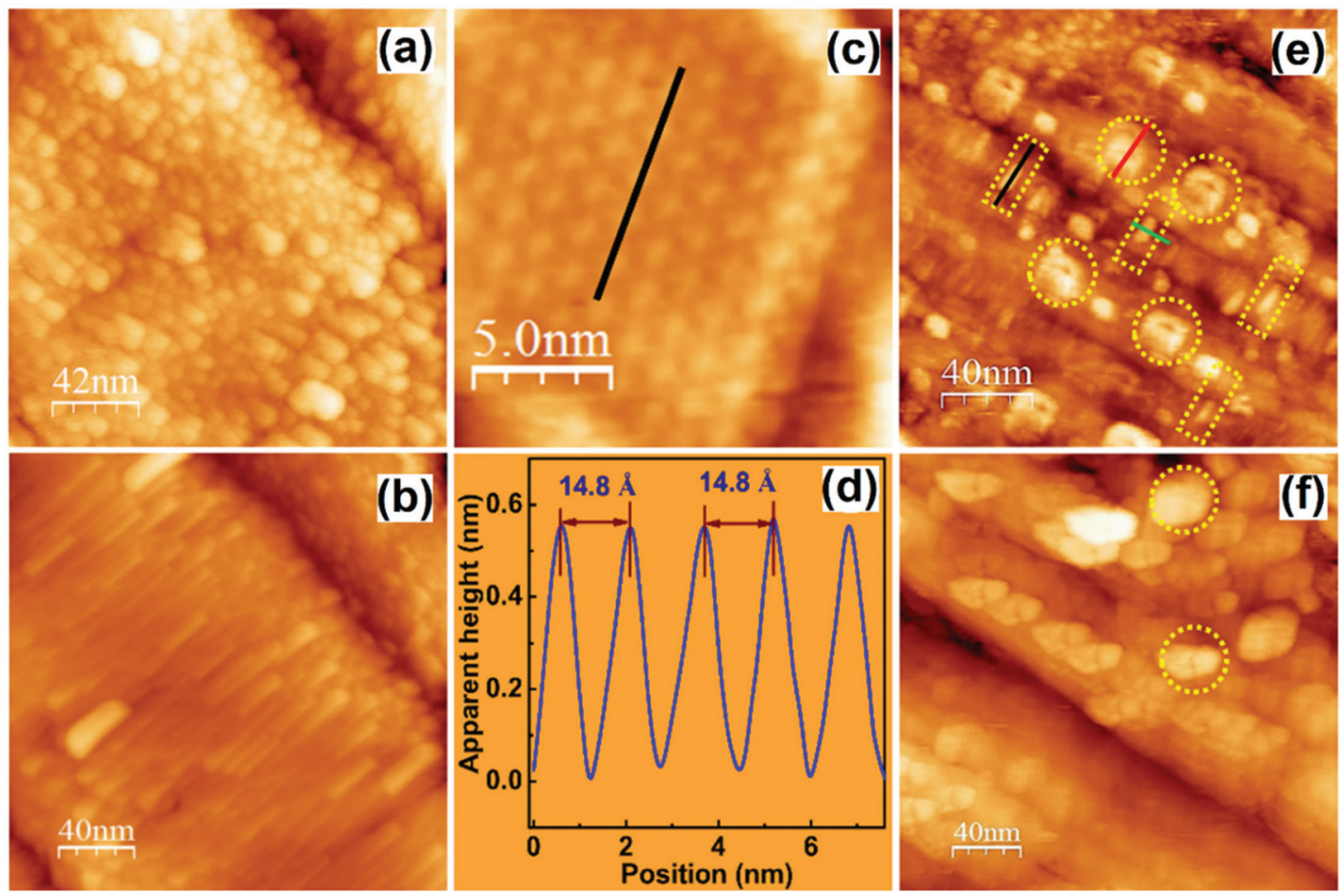

Fig. 8 (a) Aged surfaces of an as-grown single crystal; (b) the pristine surfaces of a cleaved single crystal; (c) ordered structures of the pristine surface showing well-aligned stacked planes; (d) height profile of ' $c$ ' showing the $\mathrm{Pb}-\mathrm{Pb}$ distance along the (110) plane; and (e and f) STM images of a pristine crystal showing the hydrate formation on the surface after overnight exposure to ambient air with ring-like protrusions (marked by circles) and the disunified 1D chains (marked by rectangles). (a-f) Reproduced with permission from ref. 84 . 
level, which is much closer to the VB than the CB, as shown in Fig. 9a. However, these results are in contrast to the results reported by Gallet et al., ${ }^{117}$ where the $E_{\mathrm{F}}$ level position is observed close to the $\mathrm{CB}$ than the VB, suggesting an n-type surface (Fig. 9b), supported by photoemission results reported in the studies in ref. 118 and 119. An extrapolated bandgap from the $\mathrm{d} I / \mathrm{d} V$ curve was observed at approximately $1.58 \mathrm{eV}$. In addition, the effect of surface states has been studied using an effective approach of tip-induced band bending modeled by $\mathrm{J}$. Bardeen. ${ }^{120}$ To understand the effect systematically, they have explored two different systems: (i) with no intrinsic surface states, and (ii) with a high density of surface states. The typical band schematics of an n-type semiconductor and a metallic tip are shown in Fig. 9c and d. With no applied bias, the $F_{\mathrm{E}}$ levels are lifted to the surface, since the surface states are absent (Fig. 9c). Against a positive bias applied to the tip, elec- tron accumulation occurs at the surface due to the tip-induced band bending. In short, the absence of intrinsic surface states upon the positive bias leads to amply filled localized surface states dominating the conductance in the bandgap.

On the other hand, in the case of the high density of surface states, the charge neutrality level is lower than the $E_{\mathrm{F}}$ level tending towards the negatively charged surface states, where upward band bending occurs (Fig. 9e). Under a positive bias, the tip-induced band bending does not occur due to the restrained $E_{\mathrm{F}}$ level at the surface. Fig. $9 \mathrm{~d}$ and $\mathrm{f}$ show the $\mathrm{d} I / \mathrm{d} V$ spectra of two systems with no intrinsic surface state and with high surface density states on logarithmic and linear scales. At a small negative bias, tunneling conductance is low because of the high defect density of the surface leading to strong Fermilevel pinning. Tunneling can occur from the extended states of the $\mathrm{VB}$, only when the applied bias is sufficiently negative. For (a)

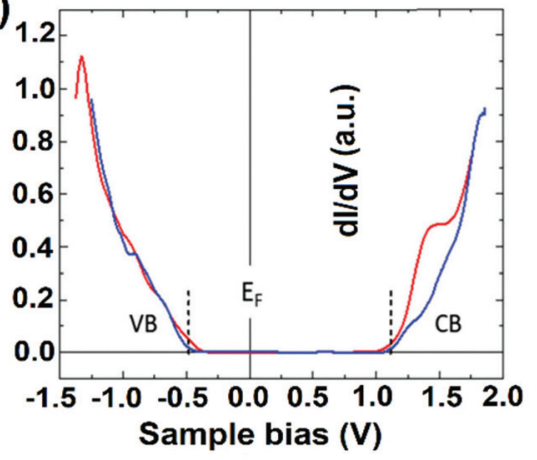

(c) Sample

Tip

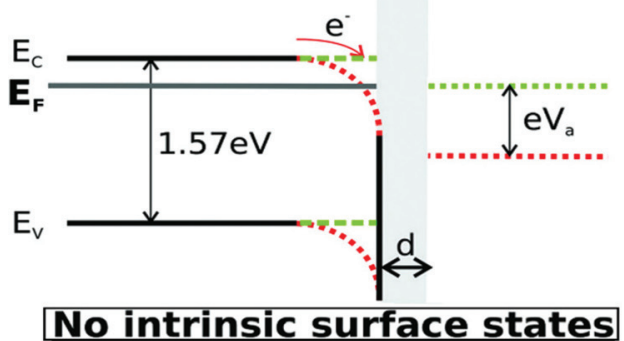

(e)

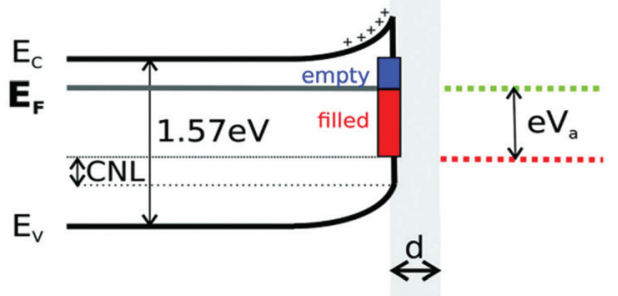

High density of surface states
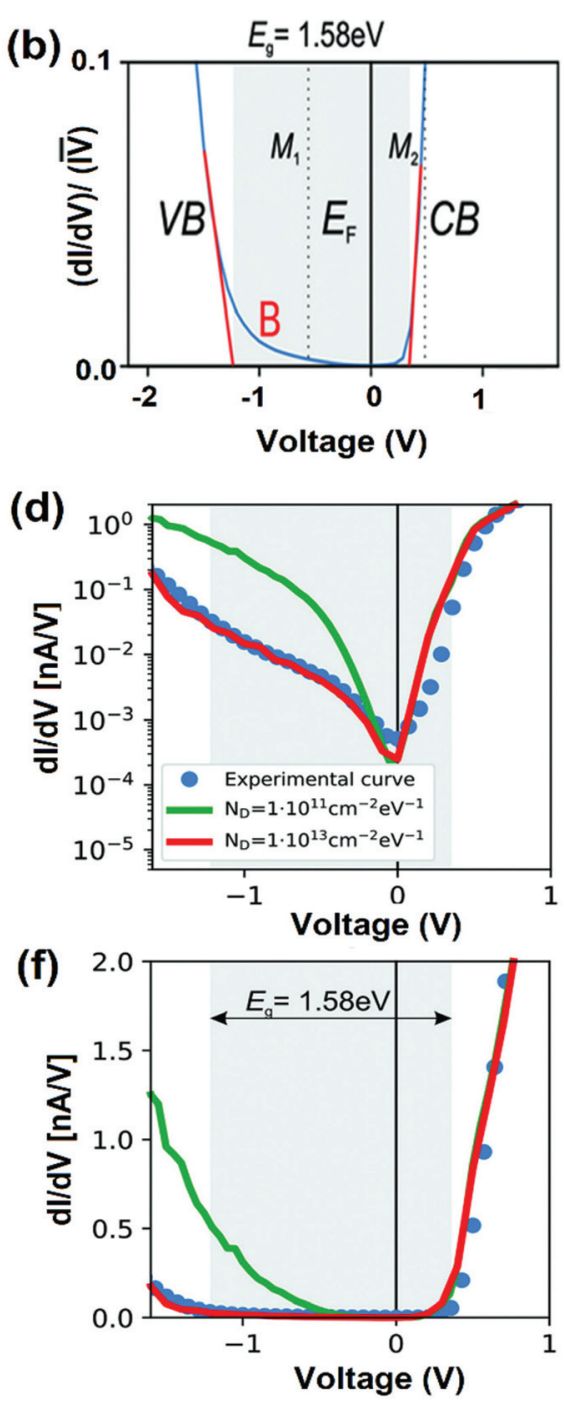

Fig. 9 (a) STS of MAPbl 3 , where the dashed lines indicate the onset of the average conduction band (CB) and valence-band (VB). The STS shows that the material is a p-type semiconductor with an energy gap of $1.53 \pm 0.05 \mathrm{eV}$. Reproduced with permission from ref. 58. (b) Averaged (dl/dV/(I-V) curve. (c and e) The sketches of the tunneling junction between the surface and the tip without (c) and with high surface density states (e). (d and f) Simulated d I/ $\mathrm{d} V$ curve on a logarithmic (d) and linear scale ( $f$ ) for the two cases represented in (c) and (e), respectively. (b-f) Reproduced with permission from ref. 117. 
these calculations, all-important parameters were considered to their highest accuracy. Such analyses are crucial, where different amounts of surface states occur due to the dissimilar surface terminations (due to exposure to air) or one type of facet that can dominate the band structure alterations.

3.1.5. Mixed halide perovskites. Using mixing halides is an alternative strategy towards making high-performance stable perovskite materials and PV devices. ${ }^{121,122}$ Different electronic phases can be obtained through halide mixing, which are accountable for altering the photo-absorption properties in halide perovskites. Two different phases have been observed by Yost et al. within the range of $10-20 \mathrm{~nm}$ size in an $\mathrm{MAPbI}_{3-x} \mathrm{Cl}_{x}$ perovskite layer due to the slight deviation of the halogen element, i.e. the $\mathrm{I} / \mathrm{Cl}$ ratio ${ }^{59}$ Nevertheless, the stabilizing effect depends on halide distribution in the mixed compounds, which is a topic of intense debate. In addition, the role of $\mathrm{Cl}$ in $\mathrm{MAPbI}_{3}$ remains a topic of discussion since many conflicting reports have been published. ${ }^{94,123,124}$ Hieulle et al. intended to solve this problem by incorporating $\mathrm{I}$ and $\mathrm{Cl}$ in the $\mathrm{MAPbBr}_{3}$ halide perovskites with an optimized concentration, but without detrimental band-gap modifications. ${ }^{125}$ Initially, $\mathrm{MAPbBr}_{3}$ perovskite deposition relies on a dual-source co-evaporation method on an atomically flat Au surface. Subsequently, $\mathrm{PbI}_{2}$ and $\mathrm{PbCl}_{2}$ are also deposited on $\mathrm{MAPbBr}_{3}$ at different temperatures to form $\mathrm{MAPbBr}_{3-y} \mathrm{I}_{y}$ and $\mathrm{MAPbBr}_{3-z} \mathrm{Cl}_{z}$, respectively. The STM image (Fig. 10a) reveals the bright spots corresponding to $\mathrm{Br}$ ions with a uniform height and width. After $\mathrm{PbI}_{2}$ deposition, a few spots become brighter as shown in Fig. 10b. However, $\mathrm{PbCl}_{2}$ deposition makes some spots slightly darker with a smaller diameter and a lower apparent height. These dark spots appear lower than the adjacent $\mathrm{Br}$ ions (Fig. 10c). Furthermore, DFT calculations have been performed to obtain exact information about the dark and bright protrusions, observed on the surface of halide perovskites by considering various cases including the adsorption of entire $\mathrm{PbI}_{2} /$ $\mathrm{PbCl}_{2}$ molecules or the substitution of $\mathrm{Br}$ by I or $\mathrm{Cl}$ ions on the surface of $\mathrm{MAPbBr}_{3}$. They observed that $\mathrm{PbI}_{2}$ (or $\mathrm{PbCl}$ ) molecules are dissociated, followed by the substitution of $\mathrm{Br}$ by I (or $\mathrm{Cl}$ ). The substitution of these molecules with a typical example of iodine is schematically shown in Fig. 10d. Hence, bright and dark protrusions are assigned to $\mathrm{I}$ and $\mathrm{Cl}$ ions in the STM images, respectively, representing the substitution of $\mathrm{Br}$ ions at the surface of halide perovskites. These results confirm the formation of $\mathrm{MAPbBr}_{3-y} \mathrm{I}_{y}$ and $\mathrm{MAPbBr}_{3-z} \mathrm{Cl}_{z}$. Furthermore, their stability has been examined to probe the impact of halide in $\mathrm{MAPbBr}_{3}$ (Fig. 10e and f). The substitution of Br by I on the surface induces a decrease in the decomposition energy (purple curve) with a small reduction in the band-gap (Fig. 10e, orange curve). In contrast, a small incorporation of Cl (below 25\%, green curve) increased the decomposition energy without a substantial change in the bandgap (Fig. 10f), which suggests the higher stability of perovskite compounds under external stimuli. Such increased stability is also attributed to the stronger bond between $\mathrm{Cl}$ and $\mathrm{Pb}$, in comparison with that of $\mathrm{Br}-\mathrm{Pb}$ and $\mathrm{I}-\mathrm{Pb}^{126}$ After $25 \%$ substitution, the incorporation of a small amount of $\mathrm{Cl}$ ions induced a strain to
$\mathrm{Br}$ resulting in lower material stability. Therefore, the stability of mixed-halide perovskites can vary due to the interaction between $\mathrm{Pb}$-halide bonds, which stabilizes the materials and generates the strain, induced by the halide substitution. As a result, the decomposition energy becomes lower. From this study, one can note that the substitution is only considered on the surface. The results also notify that there may exist an optimal substitution ratio of the bulk $\mathrm{Cl}$, which would provide higher stability without changing the bandgap of the halide perovskites. The balanced ratio of halides paves the way to resolve the stability issue of perovskite PV devices that remains a huge challenge for industrial applications.

3.1.6. Light modulated STM. The real-time charge generation and separation measurements under illumination are an important means to understand the physical phenomena in solar cells. For perovskite solar cells, C-AFM and KPFM have already been utilized to explore the photon-induced local electronic structures at the grain and grain boundaries of thinfilms. ${ }^{127-130}$ For a comprehensive understanding of charge separation and its transport behavior at the interface, Shih et al. performed STM and STS studies under specific wavelengths of light illumination. ${ }^{48}$ The important thing is that, for the first time, they observed spatially resolved mapping images of the interfacial band bending at the VB and CB upon photon illumination. This study has revealed the photogenerated carriers (electron-hole) and their transport mechanism at the heterointerfaces of $\mathrm{PbI}_{2} / \mathrm{MAPbI}_{3}$. The topography of a $\mathrm{PbI}_{2}$ passivated $\mathrm{MAPbI}_{3}$ perovskite is shown in Fig. 11a, where an approximately 200-600 nm grain size was observed. Additionally, the compositional distribution of each grain has been illustrated by the normalized differential conductance (dI/dV, Fig. 11c). One can observe from the mapping image two distinct local electronic features, more conductive at the interior (red) and less conductive at the outer side of the grain (blue), respectively. These observations suggested uneven compositional distribution at the individual grain of perovskites. To explore the band alignment in the individual grain, three grains were chosen, which were marked as 'A', 'B', and 'C' (Fig. 11c). The representative $\mathrm{d} I / \mathrm{d} V$ curve at the grain interior exhibited a more conductive feature compared with that observed at the outer part of the grain, indicating an estimated bandgap size of $1.5 \mathrm{eV}$ (Fig. 11b, upper panel). Such curves suggest the primary contribution of the $\mathrm{MAPbI}_{3}$ perovskite. In contrast, the lower panel (Fig. 11b) depicts the curve observed at the outer zone of the grain, showing less conductive features with a bandgap size of approximately $2.4 \mathrm{eV}$. This bandgap size suggests that such curves could have arisen due to the presence of $\mathrm{PbI}_{2}$ thin layers.

The mapping image of the band alignment across the perovskite/PbI ${ }_{2}$ heterointerface in the grain is shown in Fig. 11d. The VB and CB edges for $\mathrm{MAPbI}_{3}$ and $\mathrm{PbI}_{2}$ were observed around 0.3 and $0.6 \mathrm{eV}$, respectively, which subsequently proves that the band alignment is of type I heterojunction at the perovskite $/ \mathrm{PbI}_{2}$ interface. Additionally, the interior part of the $\mathrm{MAPbI}_{3}$ grain and $\mathrm{PbI}_{2}$ at the outer part of the grain behave as an intrinsic n-type semiconductor. Such STM/STS studies can 

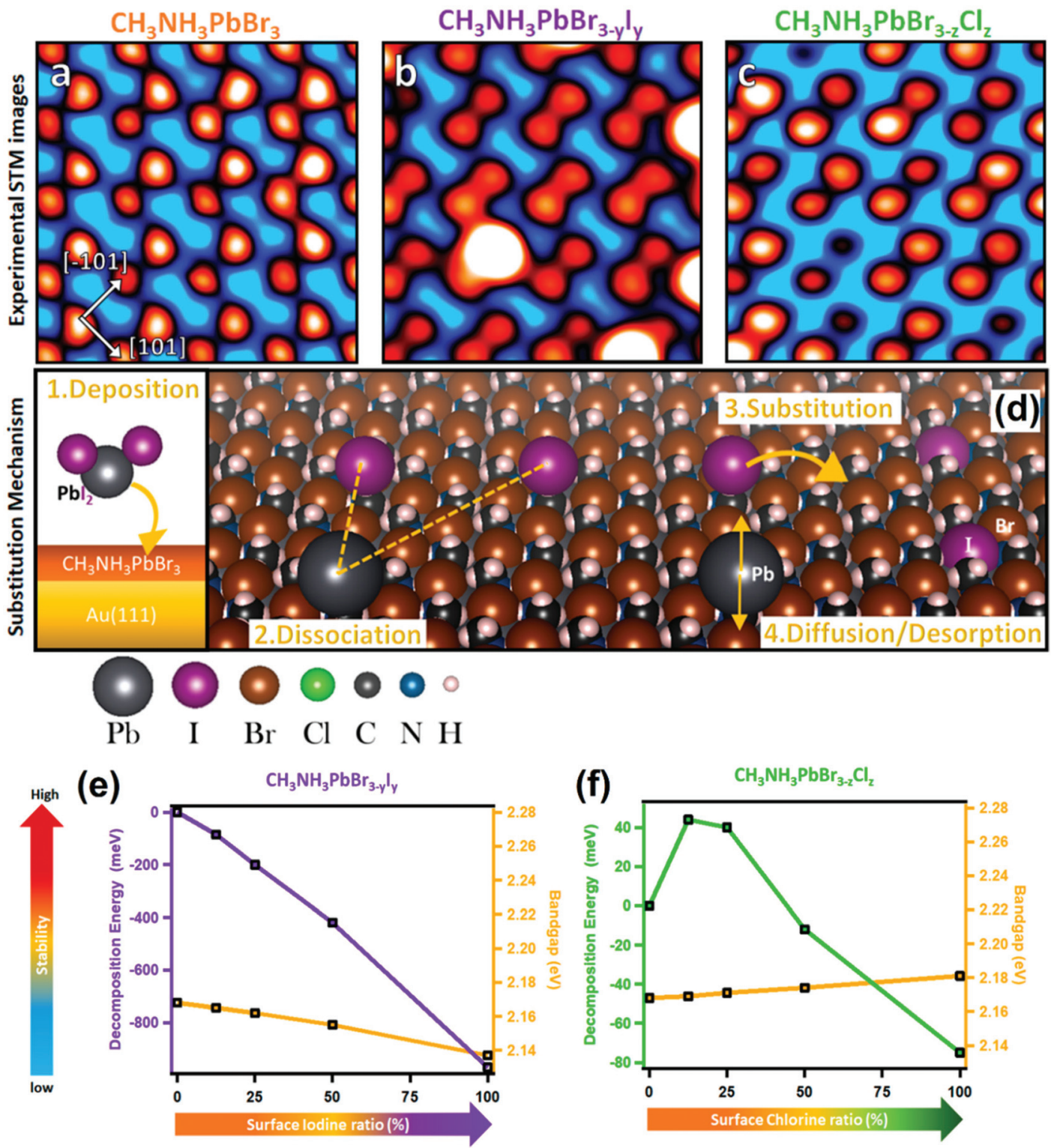

Fig. $10 \mathrm{STM}$ images of (a) $\mathrm{MAPbBr}_{3}$, (b) $\mathrm{MAPbBr}_{3-y} \mathrm{l}_{y}$, and (c) $\mathrm{MAPbBr}_{3-z} \mathrm{Cl}_{z}$ with respect to halide substitution at the perovskite surface. (d) Scheme of the substitution mechanism occurring at the surface of the $\mathrm{MAPbBr}_{3}$ perovskite after deposition of $\mathrm{Pbl}_{2}$ and $\mathrm{PbCl}_{2} \mathrm{molecules}$ (only the $\mathrm{Pbl}_{2}$ case is presented for clarity; however $\mathrm{PbCl}_{2}$ follows the same mechanism). Stability versus bandgap change in a mixed halide perovskite determined by DFT calculations: (e) MAPbBr${ }_{3-y} l_{y}$ with increasing I content at the surface, and (f) $\mathrm{MAPbBr}_{3-z} \mathrm{I}_{z}$ with increasing $\mathrm{Cl}$ content at the surface. (a-f) Reproduced with permission from ref. 125.

directly help visualize spatially resolved band alignment characteristics and electronic configurations. Furthermore, under illumination, photoinduced charge transfer and band bending were observed at the heterostructure. The shift of the energy difference between the $\mathrm{CB}$ edge $\left(E_{\mathrm{C}}\right)$ and the Fermi level $\left(E_{\mathrm{F}}\right)$, denoted as $E_{\mathrm{D}}=E_{\mathrm{C}}-E_{\mathrm{F}}$, can be used to study the photoinduced characteristics of the material. Fig. 11f exhibits the difference between the CB edges $\left(E_{\mathrm{C}}\right.$ or $\left.E_{\mathrm{C}}^{\prime}\right)$ and the Fermi level $\left(E_{\mathrm{F}}\right)$ in a dark environment and under illumination, respectively. Under light illumination, the $\mathrm{CB}$ edge $\left(E_{\mathrm{C}}\right.$, open red triangles) of the $\mathrm{MAPbI}_{3}$ part was shifted toward the higher energy $\left(E^{\prime}{ }_{C}\right.$, solid red triangular symbol). Also, light illumina- tion effects cause an increase in LDOS, as observed in the $\mathrm{d} I /$ $\mathrm{d} V$ curve at a negative sample bias. This improved LDOS effect can be seen due to the increased tunneling current from the VB to the tip, which exhibits the carrier density enhancement of photoinduced holes in the $\mathrm{MAPbI}_{3}$ parts of the perovskite grains. Fig. 11e represents a light illuminated mapping image of the spatial distribution of $\Delta E_{\mathrm{D}}$ observed for the perovskite grains, where $\Delta E_{\mathrm{D}}$ values are obtained from the variation of $E_{\mathrm{D}}$ and $E_{\mathrm{D}}^{\prime}$. This variation is observed when photoexcited carriers are generated and are separated in the perovskite crystals under illumination. $\Delta E_{\mathrm{D}}$ values in grain interiors were mostly positive (red or orange) and were either negative (blue) or 

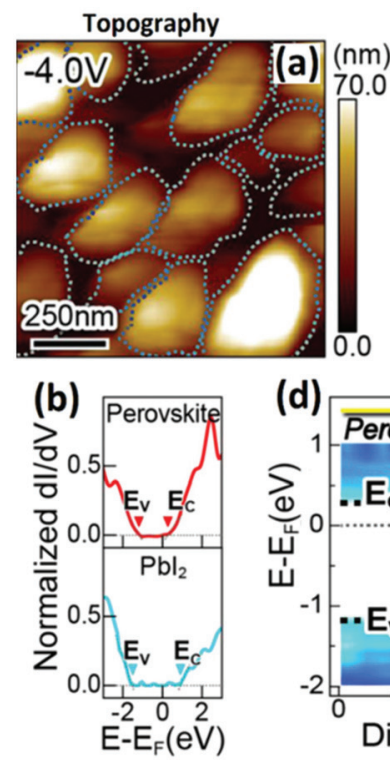

(d)
Normalized dI/dV Image
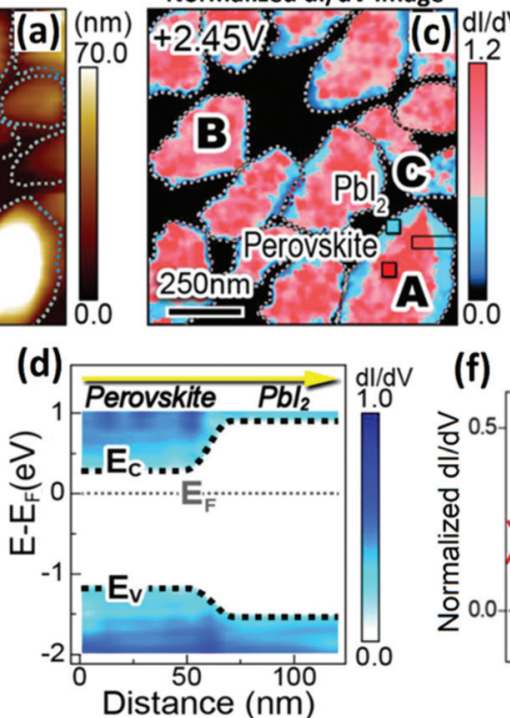

$\Delta E_{0}$ (Illumination)

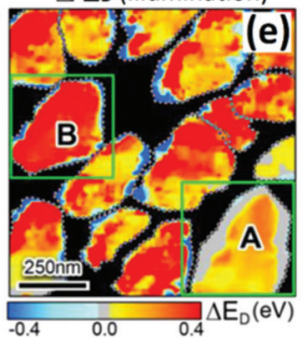

(f)

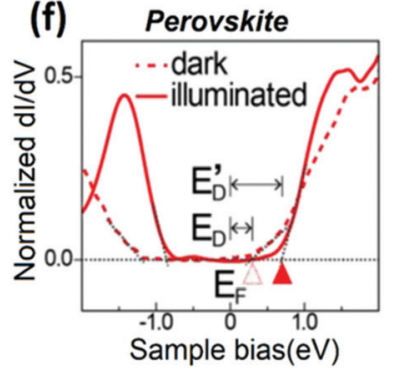

(g)

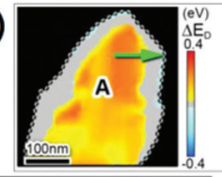

(h)
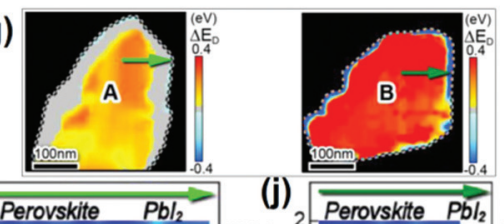

(j)

Perovskite $\mathrm{Pb} / 2$ $\mathrm{E}_{\mathrm{c}} \ldots$ $E_{v}$

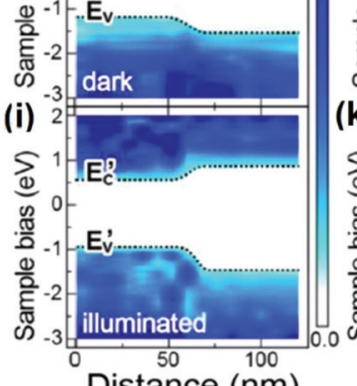

Distance (nm)

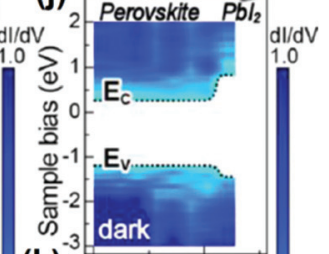

Fig. 11 (a) Typical STM morphology image. (b) Two representative normalized d//dV images of (c) (lower and upper panels) obtained at the outer part (blue square in grain A) and interior in the grain (red square in grain A), respectively. (c) A mapping image of the normalized d//dV spectra of perovskite grains recorded at a sample bias of $+2.45 \mathrm{~V}$. (d) A mapping image of the band alignment across the $\mathrm{MAPbl}_{3} / \mathrm{Pbl}_{2}$ hetero-interface in grain $\mathrm{A}$ by extracting the locations of the $\mathrm{VB}$ and $\mathrm{CB}$ edges. (e) $\mathrm{A}$ spatial distribution of $\Delta E_{\mathrm{D}}$ values $\left(\Delta E_{\mathrm{D}}=E_{\mathrm{D}}^{\prime}-E\right)$ observed for the perovskite grains under light illumination. (f) Representative $\mathrm{d} / / \mathrm{d} V$ curves of perovskites in the dark (dashed curve) and under light illumination (solid curve) conditions. (g) $\Delta E_{\mathrm{D}}$ mapping of perovskite grains ' $A$ ' and ' $B$ '. (h) and (i) correspond to the band alignments across the heterojunctions of grain ' $A$ ' consisting of a thick ( 45 nm) Pbl 2 layer under darkness and light illumination, respectively. ( $\mathrm{j}$ ) and ( $\mathrm{k}$ ) correspond to the band alignments across the heterojunctions of grain ' $\mathrm{B}$ ' consisting of a thin $(\sim 8 \mathrm{~nm}) \mathrm{Pbl}_{2}$ layer under darkness and light illumination, respectively. (a-h) Reproduced with permission from ref. 48.

nearly zero (gray) at the outer parts. This image typically shows the compositional distributions of $\mathrm{MAPbI}_{3}$ and $\mathrm{PbI}_{2}$ as shown in Fig. 11b. This study concludes that the chemical composition of perovskite grains plays a crucial role in the shifting of $\Delta E_{\mathrm{D}}$ values and the charge transfer behaviors. In addition, the STS measurements show the consequences of the thin $\mathrm{PbI}_{2}$ layers on photocarrier generation and the separation at the heterointerface of perovskite crystal grains. Fig. 11g shows the $\Delta E_{\mathrm{D}}$ band mapping across the perovskite/ $\mathrm{PbI}_{2}$ heterojunctions of grains 'A' and 'B', and pointed with green colored arrows. The interfacial band alignments at grains A and B are shown in Fig. 11h-k. These band mapping was derived from the characteristic curves of the point-to-point STS data under the dark and illumination at both grains. In grain ' $\mathrm{B}$ ', the $\mathrm{PbI}_{2}$ region showed a significant downward band bending, under illumination, whereas the energy shifts that occurred in the $\mathrm{PbI}_{2}$ region of grain A were not noteworthy. This result indicates that the transportation of photogenerated electrons was more efficient in the thinner layer of the $\mathrm{PbI}_{2}$ region of grain ' $\mathrm{B}$ '. On the other hand, the thick $\mathrm{PbI}_{2}$ layer in grain 'A' affects the photoinduced charge transfer and probability of carrier recombination. Consequently, under light illumination, the hole concentration increases in the inner area of grain ' $A$ ' compared to that of grain 'B'. Grain 'A' exhibited shifting of VB and $\mathrm{CB}$ edges by $\sim 0.25 \mathrm{eV}$ toward high energy (Fig. 11i); and an $\sim 0.40 \mathrm{eV}$ shift occurred in the perovskite region of grain ' $\mathrm{B}$ ' (Fig. 11k). This thickness dependent variation in the energy level shift may be due to the non-uniform decomposition of
$\mathrm{MAPbI}_{3}$ during thermal annealing. ${ }^{131}$ This study reveals the important role of the $\mathrm{PbI}_{2}$ passivation layers in the perovskite crystal grains. The unique LM-STM technique enables us to study photocarrier generation and interfacial electronic structures under the spatially resolved conditions offering great potential for exploration in PV and photochemical cells.

\subsection{Cesium based halide perovskites}

As the hybrid perovskite materials were developed for PV applications, atoms of cesium (Cs), $\mathrm{Rb}^{+}$, europium (Eu), etc. have also been introduced as cations in perovskite structures. ${ }^{132-135}$ The main goal of incorporating such metals into solar cells is not only to improve the efficiency, but also to increase the stability of the materials/device. Many groups have carried out numerous experiments on inorganic perovskites such as $\mathrm{CsPbBr}_{3},{ }^{136,137} \mathrm{Cs}_{2} \mathrm{InAgCl}_{6}{ }^{138}{ }^{13} \mathrm{Cs}_{2} \mathrm{AgBiBr}_{6},{ }^{139}$ and $\mathrm{Cs}_{2} \mathrm{TiBr}_{6} \cdot{ }^{140}$ Among them, $\mathrm{CsPbBr}_{3}$ is known as a highly defect tolerant material in terms of its electronic structure, and the secret of perovskite stability lies in the structure. ${ }^{141}$ Most of the inherent defects lead to shallow transition levels, though a few of them may create deep transition levels with greater formation energies. Hence, $\mathrm{CsPbBr}_{3}$ can maintain its good electronic quality despite the presence of defects.

3.2.1. Reconstruction of $\mathrm{CsPbBr}_{3}$. The evaluation of the electronic structure experimentally has not been fully investigated. Theoretically, Kang et al. have studied the charge transition levels and formation energies of intrinsic defects in $\mathrm{CsPbBr}_{3}{ }^{141}$ Recently, Hieulle et al., demonstrated the 


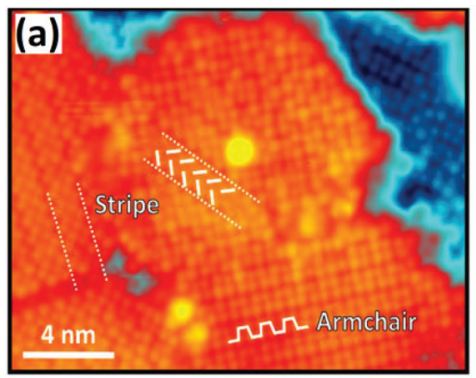

(d)

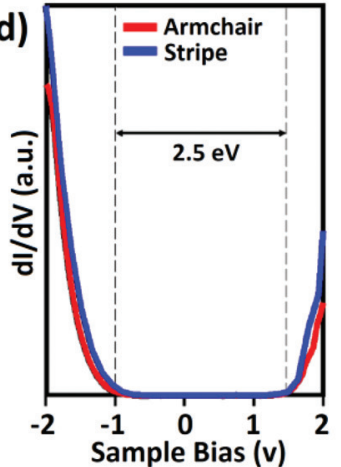

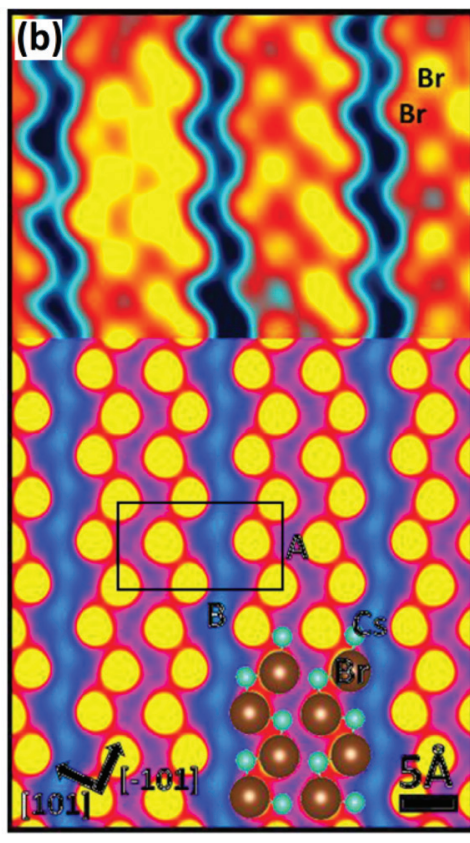

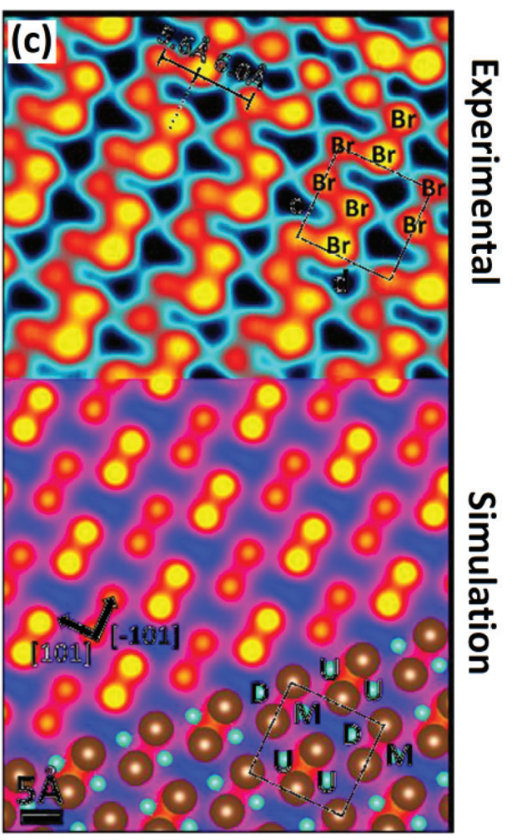

Fig. 12 (a) Topographic image of the $\mathrm{CsPbBr}_{3}$ film obtained by STM: a "stripe" domain and an "armchair" domain indicated by the white segments; experimental image and simulated model of STM. (b) The stripe reconstruction of $\mathrm{CsPbBr}_{3}$ and (c) the armchair reconstruction of $\mathrm{CsPbBr}_{3}$. The black rectangle in ' $b$ ' indicates the unit-cell of the stripe structure. The black square in 'c' represents the unit-cell of the armchair structure. (d) Local band structure determination of the $\mathrm{CsPbBr}_{3}$ perovskite for the stripe and armchair domains. (a-d) Reproduced with permission from ref. 142.

atomic structure and other electronic properties in a $\mathrm{CsPbBr}_{3}$ perovskite using STM, revealing its detailed stability mechanism. ${ }^{142}$ An atomic-resolution STM image (Fig. 12a) reveals that the $\mathrm{CsPbBr}_{3}$ surface is composed of two different reconstructions, namely a "stripe" and an "armchair" domain depending upon the Br-pair periodic arrangement. The boundary between these domains is resolved. One can observe several defects at the domain boundary, though only a few domains are nearly free from the defects. DFT calculations have been performed based on the first-principles to understand the origin of two dissimilarities in the domain, and their results have been correlated with the experimental observations, which are shown in Fig. 12b and c. The numerical results matched well with the experimental results for the (010) $\mathrm{CsPbBr}_{3}$ surface containing only $\mathrm{Cs}$ and $\mathrm{Br}$ ions. The bright protrusions in the STM images are associated with the $\mathrm{Br}$ ions; however the Cs ions were not seen owing to their lower DOS compared to $\mathrm{Br}$ ions.

The DFT model shows the evaluation of the $\mathrm{CsPbBr}_{3}$ stripe domain reconstruction, which occurred due to the alternation of the short $(6.2 \AA)$ and long (7.8 $⿱$ ) spacing between the $\mathrm{Br}$ pairs (Fig. 12b). These calculations have disclosed the formation of $\mathrm{Br}$ pairs due to the coulombic interaction between the $\mathrm{Br}$ ion and two nearby Cs cations. In contrast, the reconstruction of the armchair domain (Fig. 12c) arises from the alternation of bright and dark $\mathrm{Br}$ pair rows that originates from a change in the vertical position of surface ions. The armchair unit cell consists of four $\mathrm{Br}$ anions and four Cs cations, and is similar to the stripe domain shown in Fig. 12c (black square). Furthermore, it demonstrated that the armchair domain is less energetically favorable than the stripe domain that has a more compact nature. For both cases, DFT calculation results matched well with the experimental results. The electronic properties (STS) of the $\mathrm{CsPbBr}_{3}$ perovskite were determined independently in the stripe and armchair domains, as shown in Fig. 12d. Interestingly, the electronic properties for the stripe and armchair domains are quite similar irrespective of their significant structural differences. The evaluation of two surface structures due to the complex coulombic interactions between $\mathrm{Cs}$ and $\mathrm{Br}$ in $\mathrm{CsPbBr}_{3}$ could pave the way for understanding the stability deeply. Similarly, accurate band structure resolution could allow better prediction of interfacial properties.

\subsection{Oxide perovskites}

Oxide perovskite materials exhibit a wide range of attractive properties including superconducting, ferroelectric, magnetoresistive, and PV properties due to their structural and compositional flexibility. ${ }^{100,143}$ This section is constructed within the scope of local morphological and spectroscopic information of oxide perovskite materials such as $\mathrm{BaTiO}_{3}, \mathrm{BiFeO}_{3}, \mathrm{~Pb}(\mathrm{Zr}, \mathrm{Ti})$ $\mathrm{O}_{3}$, etc., which are actually being used as a photo-active or charge-transport layer in PV devices. ${ }^{144-149}$ Such perovskite materials possess peculiar ferroelectric properties, which can provide an effective driving force for charge generation and 
separation due to the strong intrinsic dipoles. Numerous theories have been proposed for probing the origin of this intriguing phenomenon. However, as far as the PV effect is concerned, it is difficult to figure out a single unified theory that can explain oxide perovskites with various forms. ${ }^{150,151}$

Recently, $\mathrm{Bi}_{2} \mathrm{FeCrO}_{6}$-based heterojunction devices have been demonstrated to reach $2 \%$ photoconversion efficiency (PCE). ${ }^{152}$ Similarly, several groups have performed numerous experiments on solar cells based on oxide perovskites such as $\mathrm{BiFeO}_{3},{ }^{153} \mathrm{~Pb}(\mathrm{Zr}, \mathrm{Ti}) \mathrm{O}_{3}(\mathrm{PZT}),{ }^{154}$ and $\mathrm{BaTiO}_{3}{ }^{155}$; however, all of them have shown very low PCE. Low PCEs are presumably due to the very low short-circuit current-density $\left(J_{\mathrm{sc}}\right)$ in the range of $\mu \mathrm{A}-\mathrm{mA} \mathrm{cm}^{-2}$, which cannot be determined by the large bandgap of absorbers. For instance, $\mathrm{BiFeO}_{3}$ has a relatively small bandgap, and shows absorption only in the visible region. ${ }^{156}$ However, other $\mathrm{BiFeO}_{3}$-based perovskite devices possess a relatively larger bandgap, exhibiting $J_{\text {sc }}$ in the range of 1-1.5 $\mathrm{mAcm}^{-2}$. $^{147}$ Therefore, the efficiency enhancement in oxide perovskite-based devices is a difficult and challenging arena to work upon. Furthermore, researchers are still sorting out challenges by analyzing local energy levels along with the charge transfer phenomenon and morphology. The electronic structure of a $\mathrm{SrTiO}_{3}$ perovskite has been visualized by Hitosugi et al. ${ }^{157}$ and its PV performance is demonstrated by Wu et al. ${ }^{158}$ The defect states owing to oxygen vacancies have been observed in the synthesized perovskite through the spatial distribution in electronic structures. These results can be useful to predict the performance of the device, since mostly defect states are responsible for hysteresis generation in the $I-V$ characteristics of solar cells that affect its overall performance. Cai et al. have identified the temperature-dependent properties in $\mathrm{a}_{0.8} \mathrm{Sr}_{0.2} \mathrm{CoO}_{3}$ (LSCO) perovskite from surface electronic structures, where the transition occurred from the semiconducting to metallic states. ${ }^{159}$ In brief, they explored the influence of temperature and lattice strain on the surface electronic structure. Both the tensile and compressive strained LSCO (on $\mathrm{SrTiO}_{3}$ crystal) surfaces can alter a semiconducting state (at room temperature) to a metal-like state (at elevated temperatures) captured by in situ STS.

3.3.1. $\mathbf{p}-\mathbf{i}-\mathbf{n}$ configured heterostructure. The appraisal of charge transport measurements for a PV material can be done by studying the band alignment through the tunneling current under tunneling spectroscopy. Recently, the intrinsic layer of a $\mathrm{BiFeO}_{3}$ perovskite (a photo-absorber layer) has been sandwiched between p-type $\mathrm{NiO} / \mathrm{MgO}$ and n-type $\mathrm{ZnO}$ for PV applications. ${ }^{160}$ For the confirmation of successful formation of a $\mathrm{p}-\mathrm{i}-\mathrm{n}$ configured $\mathrm{NiO}-\mathrm{BiFeO}_{3}-\mathrm{ZnO}$ and $\mathrm{MoO}_{3}-\mathrm{BiFeO}_{3}-\mathrm{ZnO}$ heterojunction with a type-II band alignment at the two interfaces, Chatterjee et al. have located the CB and VB by recording the tunneling current versus tip voltage characteristics of ultrathin films. ${ }^{160}$ Fig. 13a-d exhibit the DOS of a NiO thin film, and $\mathrm{MoO}_{3}, \mathrm{BiFeO}_{3}$, and $\mathrm{ZnO}$ nanoparticles (NPs), calculated from the tunneling current. The tip voltage has been applied with respect to the substrate; the analysis of the peaks in the DOS spectra shows CB and VB edges of the semiconductors. When a positive voltage was applied to the tip, the tip with- drew electrons from the semiconductor; this indicated the VB of the semiconductor. Similarly, electrons were injected into the semiconductor, when negative voltages were applied to the tip, which was denoted as the CB. In other words, this study has shown and followed standard STM, which states that the VB lies in the negative bias of the sample.

In the DOS spectra, the valence band's position with respect to Fermi energy decided the p-type nature of $\mathrm{NiO}$ and $\mathrm{MoO}_{3}$ (Fig. 13a and b), and the n-type nature of $\mathrm{ZnO}$ (Fig. 13d) nanocrystal semiconductors. The DOS spectrum of $\mathrm{BiFeO}_{3} \mathrm{NPS}$ showed an intrinsic semiconductor characteristic (Fig. 13c). The detailed analysis of the band edges of $\mathrm{NiO}, \mathrm{MoO}_{3}, \mathrm{BiFeO}_{3}$, and ZnO NPs showed both the developed structures, i.e. NiO$\mathrm{BiFeO}_{3}-\mathrm{ZnO}$ and $\mathrm{MoO}_{3}-\mathrm{BiFeO}_{3}-\mathrm{ZnO}$ heterojunctions would form $\mathrm{p}-\mathrm{i}-\mathrm{n}$ structures with a type-II band alignment at both the interfaces (Fig. 13e and f). These kinds of heterojunctions can further be very efficient at quick charge separation within their charge carrier lifetime when used in PV materials. Subsequently, these materials can become proficient in the PV arena.

\subsubsection{Morphologically defined LSMO. Non-toxic} $\mathrm{La}_{x} \mathrm{Sr}_{1-x} \mathrm{MnO}_{3}$ (LSMO) is an appealing semiconductor, and is also a member of the perovskite family, possessing several essential properties including catalytic, magnetic and electrical properties along with good thermal and chemical stability. ${ }^{161-164}$ It has been used in PV applications owing to the ease of synthesis processes and stable catalytic activity, especially in DSSCs as a counter electrode instead of platinum (Pt). ${ }^{165}$ The PCE of LSMO counter electrode-based devices reaches around $6.6 \%$, which is comparable with the efficiency of Pt electrode-based devices. The success of the electrode is mainly due to the better electronic transport properties and uniform columnar closely packed islands of perovskite materials. The actual reason for the device's better performance is its electronic properties including the conductivity at/ on the grain boundaries. ${ }^{166}$ Datar and co-workers have investigated its electronic properties and phase transition using STM/STS to understand local DOS. ${ }^{65}$ They synthesized a couple of $\mathrm{La}_{0.7} \mathrm{Sr}_{0.3} \mathrm{MnO}$ thin films using the PLD technique with different morphologies, e.g., a packed-island (sample I) and a smooth morphology (sample II). The different behaviors on the grain vis-a-vis on the grain boundary have been observed via STM imaging.

Fig. 14a and $\mathrm{b}$ exhibit the surface morphological view of sample-I and sample-II. Sample-I showed spherical and densely packed individual grains; whereas sample-II showed continuous film formation with irregular grains and their broad size distribution. Fig. $14 \mathrm{c}$ and d depict comparative $\mathrm{d} I /$ $\mathrm{d} V$ curves of sample-I and sample-II on the grain and grain boundaries that generate a picture of LDOS near the Fermi energy of the samples. The difference in $\mathrm{d} I / \mathrm{d} V$ curves at two different locations (i.e. on the grain and at the grain boundaries) reflected the coexistence of conducting and insulating regions. This difference typically depended upon the position of the grain boundary and it was more prominent in sample-II than in sample-I. Sample-I showed a firm morphology and 

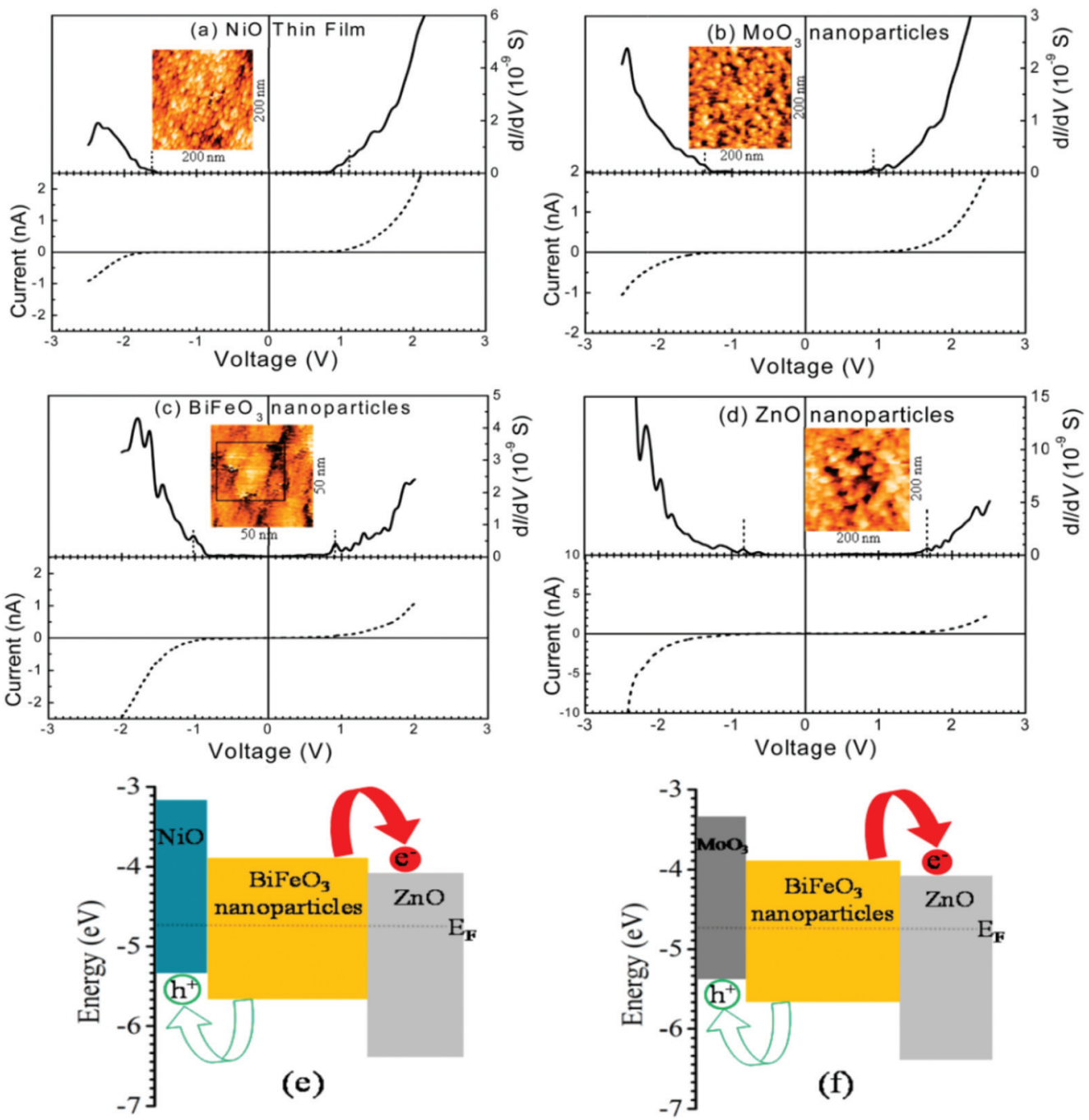

Fig. 13 Current-voltage and density of states (d//dV) plots of (a) a NiO thin film and (b) $\mathrm{MoO}_{3}$ (c) $\mathrm{BiFeO}_{3}$ and (d) ZnO nanoparticles. STM topography of the active layer is shown in the inset of each figure. Schematic description of the energy level diagrams of (e) $\mathrm{NiO}_{-}-\mathrm{BiFeO}_{3}-\mathrm{ZnO}$ and (f) $\mathrm{MoO}_{3}-$ $\mathrm{BiFeO}_{3}-\mathrm{ZnO}$ heterojunctions. (a-f) Reproduced with permission from ref. 160.

hence the presence of more systematic semiconducting regions. In this finding, two types of typical characteristics of both films were studied. Firstly, a linear region at the Fermi energy, corresponding to the metallic phase, and other plateau regions that signify the presence of a semiconducting gap in both samples were observed. Sample-II showed a small shift in the pseudogap as compared to sample-I. However, the grain boundary defines the presence of a semiconducting gap. In comparison with sample-I, the gap increases in sample-II. Sample-I showed more prominent grain boundary distribution, where this kind of semiconducting grain boundary can be well situated for device applications.

LCMO $\left(\mathrm{La}_{x} \mathrm{Ca}_{1-x} \mathrm{MnO}_{3}\right)$ also presents some PV properties with different heterostructures studied by various groups. ${ }^{167-170}$ Wei et al. have demonstrated the PV effect in $\mathrm{La}_{0.67} \mathrm{Ca}_{0.33} \mathrm{MnO}_{3} / \mathrm{LaMnO}_{3} / \mathrm{Nb}: \mathrm{SrTiO}_{3}$ hetero-junctions, where the PV response has been observed to improve significantly as the film thickness increases from 5 to $50 \mathrm{~nm}$ (saturates above
$50 \mathrm{~nm}$ ), and $\mathrm{Ni}$ et al. investigated the transient PV properties of $\mathrm{La}_{0.4} \mathrm{Ca}_{0.6} \mathrm{MnO}_{3} / \mathrm{Nb}: \mathrm{SrTiO}_{3}$ hetero-junctions for a wide range of temperatures from 293 to $723 \mathrm{~K}^{168}$ They observed that the oxygen vacancies may exist in LCMO thin films during deposition at different temperatures, leading to the variation in the $I-V$ characteristics. Furthermore, they have explained the transient photo-response with the help of an LCMO/NSTO junction under applied biases. Upon applying a positive bias to the LCMO thin films, positively charged oxygen vacancies transfer from LCMO to the LCMO/NSTO interface and form an interface barrier. Near the interface, highly oxygen-deficient LCMO experiences a wide bandgap that can be an effective barrier for the conduction of charge carriers. The surface oxygen stoichiometry in LCMO has been studied in detail via STM/STS to obtain more insights into both chemical (e.g. surface electrochemistry) and physical (e.g. LDOS, band structure, etc.) properties. ${ }^{171} \mathrm{La}_{0.625} \mathrm{Ca}_{0.375} \mathrm{MnO}_{3}$ thin films on SrTiO (001) have also been investigated through in situ STM, in a real-time 

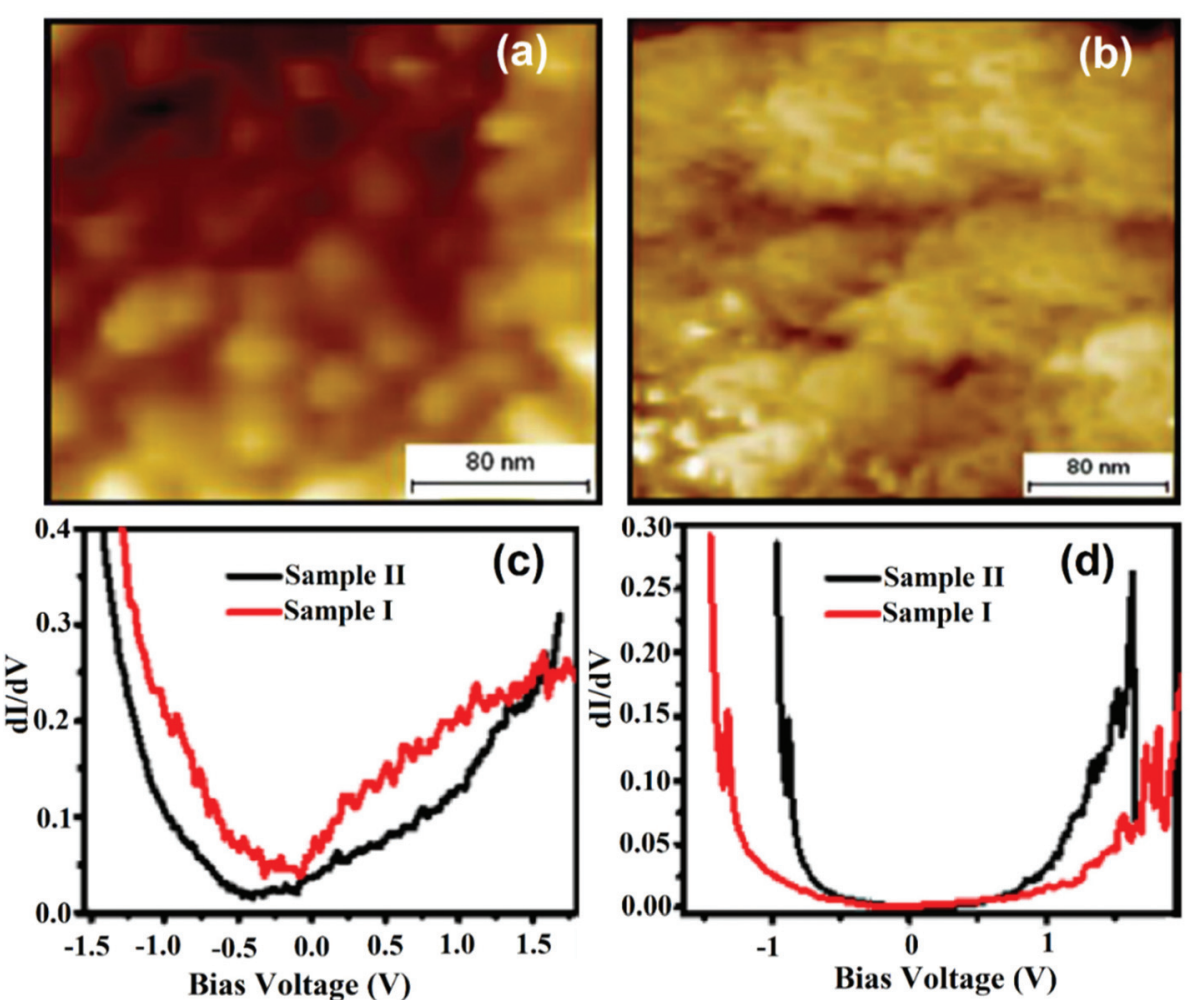

Fig. 14 Recorded topographic STM images of (a) sample-I: La $\mathrm{D}_{0.7} \mathrm{Sr}_{0.3} \mathrm{MnO}$ with a packed-island and (b) sample-II: La $\mathrm{L}_{0.7} \mathrm{Sr}_{0.3} \mathrm{MnO}$ with a smooth morphology; comparison of $\mathrm{d} / / \mathrm{d} V$ of sample-I and sample-II (c) on the grain and (d) on the grain boundary. (a-d) Reproduced with permission from ref. 65 .

fashion. They demonstrated the ability to form oxygen vacancies along with the removal and deposition of individual atomic units under different potential stresses. These studies can help us understand the surface reactions in perovskite systems.

\section{Conclusion and outlook}

The morphology and local electronic properties of the materials are highly correlated with their relevant physical, chemical, and electronic parameters, which in turn affect the device performance, for example of solar cells. While traditional imaging techniques such as SEM, AFM, and TEM can offer excellent information about the quality, roughness, and thickness of thin films, all of them lack the ability to identify different materials that in general are binary components in solar cells. In this regard, STM/STS based on its unique operation principle presents the powerful ability in terms of many aspects. With STS/STS, the electronic states of materials can be readily analyzed at nanoscale precision. For example: (i) the band diagram of solar cells can be drawn based on the aspect of charge carriers in a device; (ii) the band alignment in a junction of a PV device can be drawn before actually incorporating into a device; and (iii) the $\mathrm{d} I / \mathrm{d} V$ function in STM/STS can reveal the energetic mapping domains of the heterojunction of the energy maps of different materials. KPFM is basically an
AFM mode, and functions in the similar way of STM/STS. However, STM/STS obtains electronic spectroscopy from the LDOS for the target materials presenting a relatively much more straightforward manner in analytical propose. Besides it, the tips of STM are easier to prepare compared with those of AFM (e.g., by just mechanically cutting a metal wire, typically either Pt/Ir or tungsten).

As mentioned earlier, we have witnessed the unprecedented power of STM/STS in analyses of energy materials. We have thus reviewed recent examples to strengthen the basic understanding of the structural studies of both oxide and halide perovskite materials by using the STM/STS technique in conjunction with their related applications in solar cells. In particular, STM/STS among other analytical tools stands out as a complementary means to unveil the unique information of materials at the atomic and electronic levels. It allows in situ measurements capable of real-time monitoring of the growth and evolution of materials-formation processes. A sample with an atomically flat surface is a requirement in STM analysis, facilitating in-depth and clear electronic information. Different deposition techniques lead to the formation of surface defects in a crystal or during thin-film formation. As reviewed, the cleaving method is also known as X-STM, which has been used for sample preparation from single-crystals for STM observations. In addition, samples can also be deposited through spin coating and CVD techniques. Sometimes, the samples prepared by spin coating and CVD techniques may need 
further processes using a cleaving method, subject to the requirement of the sample for the cross-sectional interfacial study.

The STM/STS techniques can provide an in-depth understanding of the stability, charge carrier dynamics, and other optoelectronic conversion-related properties at heterojunction interfaces of materials. For the halide perovskites, STM/STS studies of the $\mathrm{MAPbBr}_{3}$ electronic structure reveal the contribution of $\mathrm{Br}$ and MA orbitals for the electron density states on the perovskite surface along with the real-time view of dislocation and intrinsic defects in a single crystal. Such results are crucial for understanding the behavior of the halide perovskite surfaces at the atomic scale, where the orientation of MA modifies the dipole strength and hence the stability of the structure. The various kinds of defects such as, cationic, anionic, excess halide, and antisite defects on the perovskite surface affect the band structure and the local work function, which will further alter the charge carrier dynamics, lifetime and subsequent recombination at the interface of the heterojunctions. Such surface and energy levels related to systematic STM/STS analysis can directly correlate the atomic interface phenomenon with the device performance from the perspective of defects and interface engineering.

The mixing of halides provides stability to the hybrid perovskites under specific moisture, temperature, and light conditions. For example, the amount of variation in I and $\mathrm{Cl}$ concentrations as a part of $\mathrm{MAPbBr}_{3}$ depicts the stability without a destructive band gap alteration, which further provides a conclusive direction for the fabrication of stable and efficient hybrid perovskite PV devices. These studies offer convincing responses to the current discussion in the world energy forum on halide variation or incorporation in hybrid perovskites for durable large-scale devices. $\mathrm{CsPbBr}_{3}$ has become a novel halide perovskite material in the PV domain due to its nearly defect-free nature and improved stability. Even structurally different forms of $\mathrm{CsPbBr}_{3}$ also show quite similar electronic properties. Furthermore, this tool has been used to explore oxide perovskites that bear ferroelectric properties, believed to carry dynamic force for the charge carrier generation and extraction mechanism due to the strong intrinsic dipole interactions. The rapid movement of charges enhances the opencircuit voltage in the PV devices, and hence the overall performance. These literature reports provide important information regarding the stability improvement of the materials and the efficiency enhancement of the corresponding devices, based on their structural properties such as compositions, defects, vacancies, and terminations of the structure. Upon studying these structural properties, one can follow a similar theoretical approach for studying an atom experimentally to its utmost depth. To this end, STM stands out as the best technique. Along this direction, further research efforts should focus on defect tolerance and its origins, which will help researchers design new materials with better defect tolerance, higher stability, and minimal toxicity. We expect that the in situ atomic and electronic visualizations of the real-time sample preparation and their time-dependent evolution through STM works will pave the way for uncovering numerous crucial and puzzling phenomena in oxide and halide perovskites, such as ion/charge carrier migration, hysteresis, and the stability mechanism, eventually enabling commercialization of PVs soon.

\section{Conflicts of interest}

There are no conflicts to declare.

\section{Acknowledgements}

The authors thank NSFC (21972095), Shenzhen University, Guangdong government (2018A030313467), Shenzhen city (1. the overseas talent set-up funding; 2. exploring multicomponent supramolecular systems, JCYJ20180305124732178; and 3. Shenzhen University and National Taipei University of Technology joint funding (2019008)). China's Postdoctoral Science Foundation (65th Batch-2019M653033). The authors thank Prof. Steven De Feyter (K. U. Leuven) for his grateful discussion with us in this review.

\section{References}

1 W. Q. Wu, Q. Wang, Y. Fang, Y. Shao, S. Tang, Y. Deng, H. Lu, Y. Liu, T. Li, Z. Yang and A. Gruverman, Nat. Commun., 2018, 9, 1625.

2 D. Zhao, C. Wang, Z. Song, Y. Yu, C. Chen, X. Zhao, K. Zhu and Y. Yan, ACS Energy Lett., 2018, 3, 305.

3 D. W. De Quilettes, K. Frohna, D. Emin, T. Kirchartz, V. Bulovic, D. S. Ginger and S. D. Stranks, Chem. Rev., 2019, 119, 11007.

4 J. Shi, Y. Wang and Y. Zhao, Energy Environ. Mater., 2019, 2, 73.

5 R. Lin, K. Xiao, Z. Qin, Q. Han, C. Zhang, M. Wei, M. I. Saidaminov, Y. Gao, J. Xu, M. Xiao and A. Li, Nat. Energy, 2019, 4, 864.

6 S. A. Veldhuis, P. P. Boix, N. Yantara, M. Li, T. C. Sum, N. Mathews and S. G. Mhaisalkar, Adv. Mater., 2016, 28, 6804.

7 C. Li, S. Cong, Z. Tian, Y. Song, L. Yu, C. Lu, Y. Shao, J. Li, G. Zou, M. H. Rümmeli and S. Dou, Nano Energy, 2019, 60, 247.

8 X. Yang, L. F. Ma and D. Yan, Chem. Sci., 2019, 10, 4567.

9 J. Lu, X. Lin, X. Jiao, T. Gengenbach, A. D. Scully, L. Jiang, B. Tan, J. Sun, B. Li, N. Pai and U. Bach, Energy Environ. Sci., 2018, 11, 1880.

10 B. Parida, S. Yoon, S. M. Jeong, J. S. Cho, J. K. Kim and D. W. Kang, Sol. Energy Mater. Sol. Cells, 2020, 204, 110212.

11 Q. Wali, F. J. Iftikhar, N. K. Elumalai, Y. Iqbal, S. Yousaf, S. Iqbal and R. Jose, Curr. Appl. Phys., 2020, 20, 720.

12 Z. Wang, Y. Gu, J. Qi, S. Lu, P. Li, P. Lin and Y. Zhang, RSC $A d v ., 2015, \mathbf{5}, 42075$. 
13 P. L. Qin, H. W. Lei, X. L. Zheng, Q. Liu, H. Tao, G. Yang, W. J. Ke, L. B. Xiong, M. C. Qin, X. Z. Zhao and G. J. Fang, Adv. Mater. Interfaces, 2016, 3, 1500799.

14 B. Yang, C. C. Brown, J. Huang, L. Collins, X. Sang, R. R. Unocic, S. Jesse, S. V. Kalinin, A. Belianinov, J. Jakowski and D. B. Geohegan, Adv. Funct. Mater., 2017, 27, 1700749.

15 T. Bu, X. Liu, Y. Zhou, J. Yi, X. Huang, L. Luo, J. Xiao, Z. Ku, Y. Peng, F. Huang and Y. B. Cheng, Energy Environ. Sci., 2017, 10, 2509.

16 C. Li, S. Tscheuschner, F. Paulus, P. E. Hopkinson, J. Kießling, A. Köhler, Y. Vaynzof and S. Huettner, Iodine migration and its effect on hysteresis in perovskite solar cells, Adv. Mater., 2016, 28, 2446.

17 T. S. Sherkar, C. Momblona, L. Gil-Escrig, J. ávila, M. Sessolo, H. J. Bolink and L. J. A. Koster, ACS Energy Lett., 2017, 2, 1214.

18 J. H. Lee, J. Kim, G. Kim, D. Shin, S. Y. Jeong, J. Lee, S. Hong, J. W. Choi, C. L. Lee, H. Kim and Y. Yi, Energy Environ. Sci., 2018, 11, 1742.

19 J. Kim, B. W. Park, J. Baek, J. S. Yun, H. W. Kwon, J. Seidel, H. Min, S. Coelho, S. Lim, S. Huang and K. Gaus, J. Am. Chem. Soc., 2020, 142, 6251.

20 S. A. Kulkarni, S. G. Mhaisalkar, N. Mathews and P. P. Boix, Small, 2019, 3, 1800231.

21 M. Saliba, J. P. Correa-Baena, M. Grätzel, A. Hagfeldt and A. Abate, Angew. Chem., Int. Ed., 2018, 57, 2554.

22 M. Long, T. Zhang, M. Liu, Z. Chen, C. Wang, W. Xie, F. Xie, J. Chen, G. Li and J. Xu, Adv. Mater., 2018, 30, 1801562.

23 X. Zhu, J. Lee and W. D. Lu, Adv. Mater., 2017, 29, 1700527.

24 X. Zheng, Y. Deng, B. Chen, H. Wei, X. Xiao, Y. Fang, Y. Lin, Z. Yu, Y. Liu, Q. Wang and J. Huang, Adv. Mater., 2018, 30, 1803428.

25 M. Chen, M. G. Ju, H. F. Garces, A. D. Carl, L. K. Ono, Z. Hawash, Y. Zhang, T. Shen, Y. Qi, R. L. Grimm and D. Pacifici, Nat. Commun., 2019, 10, 16.

26 M. B. Islam, M. Yanagida, Y. Shirai, Y. Nabetani and K. Miyano, Sol. Energy Mater. Sol. Cells, 2019, 195, 323.

27 Y. J. Chen, M. J. Zhang, S. Yuan, Y. Qiu, X. B. Wang, X. Jiang, Z. Gao, Y. Lin and F. Pan, Nano Energy, 2017, 36, 303.

28 C. S. Kley, C. Dette, G. Rinke, C. E. Patrick, J. Čechal, S. J. Jung, M. Baur, M. Dürr, S. Rauschenbach, F. Giustino and S. Stepanow, Nano Lett., 2014, 14, 563.

29 S. Rößler, B. Padmanabhan, S. Elizabeth, H. L. Bhat, F. Steglich and S. Wirth, Appl. Phys. Lett., 2010, 96, 202512.

30 Y. Almadori, D. Moerman, J. L. Martinez, P. Leclère and B. Grévin, Beilstein J. Nanotechnol., 2018, 9, 1695.

31 C. Wang, C. Zhang, S. Tong, J. Shen, C. Wang, Y. Li, S. Xiao, J. He, J. Zhang, Y. Gao and J. Yang, J. Phys. Chem. C, 2017, 121, 6575.

32 J. J. Li, J. Y. Ma, Q. Q. Ge, J. S. Hu, D. Wang and L. J. Wan, ACS Appl. Mater. Interfaces, 2015, 7, 28518.
33 R. Kumar, D. Varandani and B. R. Mehta, Carbon, 2016, 98, 41.

34 B. Huang, G. Kong, E. N. Esfahani, S. Chen, Q. Li, J. Yu, N. Xu, Y. Zhang, S. Xie, H. Wen and P. Gao, npj Quantum Mater., 2018, 3, 30.

35 M. Cai, N. Ishida, X. Li, X. Yang, T. Noda, Y. Wu, F. Xie, H. Naito, D. Fujita and L. Han, Joule, 2018, 2, 296.

36 T. Glatzel, S. Sadewasser and M. C. Lux-Steiner, Appl. Surf. Sci., 2003, 210, 84.

37 W. Li, M. U. Rothmann, A. Liu, Z. Wang, Y. Zhang, A. R. Pascoe, J. Lu, L. Jiang, Y. Chen, F. Huang and Y. Peng, Adv. Energy Mater., 2017, 7, 1700946.

38 M. M. Tavakoli, M. Saliba, P. Yadav, P. Holzhey, A. Hagfeldt, S. M. Zakeeruddin and M. Grätzel, Adv. Energy Mater., 2019, 9, 1802646.

39 D. Kim, J. H. Yun, M. Lyu, J. Kim, S. Lim, J. S. Yun, L. Wang and J. Seidel, J. Phys. Chem. C, 2019, 23, 14144.

40 S.-L. Lee, N.-T. Lin, C.-H. Chen, H.-C. Yang and T.-Y. Luh, Chem. - Eur. J., 2009, 15, 11594.

41 J. R. O’Dea, L. M. Brown, N. Hoepker, J. A. Marohn and S. Sadewasser, MRS Bull., 2012, 37, 642.

42 G. Binnig, H. Rohrer, C. Gerber and E. Weibel, Phys. Rev. Lett. , 1982, 49, 57.

43 M. Saeed, A. Mahmood, A. S. Saleemi, X. Zeng and S. L. Lee, J. Phys. Chem. C, 2020, 124, 829.

44 U. Dasgupta, A. Bera and A. J. Pal, ACS Energy Lett., 2017, 2, 582 .

45 R. Ohmann, L. K. Ono, H. S. Kim, H. Lin, M. V. Lee, Y. Li, N. G. Park and Y. Qi, J. Am. Chem. Soc., 2015, 137, 16049.

46 L. She, M. Liu and D. Zhong, Atomic structures of $\mathrm{CH}_{3} \mathrm{NH}_{3} \mathrm{PbI}_{3}$ (001) surfaces, ACS Nano, 2015, 10, 1126.

47 T. Hitosugi, R. Shimizu, T. Ohsawa, et al., Chem. Rec., 2014, 14, 935.

48 M. C. Shih, S. S. Li, C. H. Hsieh, Y. C. Wang, H. D. Yang, Y. P. Chiu, C. S. Chang and C. W. Chen, Nano Lett., 2017, 17, 1154.

49 C. Renner, G. Aeppli and H. M. Ronnow, Mater. Sci. Eng., $C, 2005,25,775$.

50 N. D. Desai, V. B. Ghanwat, K. V. Khot, S. S. Mali, C. K. Hong and P. N. Bhosale, J. Mater. Sci.: Mater. Electron., 2016, 27, 2385.

51 T. Dimopoulos, M. Bauch, R. A. Wibowo, N. Bansal, R. Hamid, M. Auer, M. Jäger and E. J. W. List-Kratochvil, Mater. Sci. Eng., B, 2015, 200, 84.

52 A. Fujiki, Y. Miyake, Y. Oshikane, M. Akai-Kasaya, A. Saito and Y. Kuwahara, Nanoscale Res. Lett., 2011, 6, 1.

53 A. Sasaki, F. Iwata, A. Katsumata, T. Akiyama and H. Fujiyasu, Rev. Sci. Instrum., 1997, 68, 1296.

54 L. She, M. Liu, X. Li, Z. Cai and D. Zhong, Surf. Sci., 2017, 656, 17.

55 M. Graetzel, R. A. Janssen, D. B. Mitzi and E. H. Sargent, Nature, 2012, 488, 304.

56 H. L. Hsu, C. P. Chen, J. Y. Chang, Y. Y. Yu and Y. K. Shen, Nanoscale, 2014, 6, 10281. 
57 L. Dou, A. B. Wong, Y. Yu, M. Lai, N. Kornienko, S. W. Eaton, A. Fu, C. G. Bischak, J. Ma, T. Ding and N. S. Ginsberg, Science, 2015, 349, 1518.

58 D. Azulay, I. Levine, S. Gupta, E. Barak-Kulbak, A. Bera, G. San, S. Simha, D. Cahen, O. Millo, G. Hodes and I. Balberg, Phys. Chem. Chem. Phys., 2018, 20, 24444.

59 A. J. Yost, A. Pimachev, C. C. Ho, S. B. Darling, L. Wang, W. F. Su, Y. Dahnovsky and T. Chien, ACS Appl. Mater. Interfaces, 2016, 8, 29110.

60 S. Wang, L. K. Ono, M. R. Leyden, Y. Kato, S. R. Raga, M. V. Lee and Y. Qi, J. Mater. Chem. A, 2015, 3, 14631.

61 M. Liu, M. B. Johnston and H. J. Snaith, Nature, 2013, 501, 395.

62 O. Malinkiewicz, A. Yella, Y. H. Lee, G. M. Espallargas, M. Graetzel, M. K. Nazeeruddin and H. J. Bolink, Nat. Photonics, 2014, 8, 128.

63 C. Stecker, K. Liu, J. Hieulle, R. Ohmann, Z. Liu, L. K. Ono, G. Wang and Y. Qi, ACS Nano, 2019, 13, 12127.

64 C. W. Chen, H. W. Kang, S. Y. Hsiao, P. F. Yang, K. M. Chiang and H. W. Lin, Adv. Mater., 2014, 26, 6647.

65 A. Joshi, R. Nori, S. Dhobale, V. R. Rao, S. N. Kale and S. Datar, Phys. B, 2014, 448, 85.

66 M. M. Tavakoli, L. L. Gu, Y. Gao, C. Reckmeier, J. He, A. L. Rogach, Y. Yao and Z. Y. Fan, Fabrication of efficient planar perovskite solar cells using a one-step chemical vapor deposition method, Sci. Rep., 2015, 5, 1408.

67 M. R. Leyden, M. V. Lee, S. R. Raga and Y. B. Qi, J. Mater. Chem. A, 2015, 3, 16097.

68 J. Ávila, C. Momblona, P. P. Boix, M. Sessolo and H. J. Bolink, Joule, 2017, 1, 431.

69 G. Q. Tong, X. Z. Lan, Z. H. Song, G. P. Li, H. Li, L. W. Yu, J. Xu, Y. Jiang, Y. Sheng, Y. Shi, et al., Mater. Today Energy, 2017, 5, 173.

70 A. Babaei, W. Soltanpoor, M. A. Tesa-Serrate, S. Yerci, M. Sessolo and H. J. Bolink, Energy Technol., 2019, 1900784.

71 L. Yang, W. L. Tsai, C. S. Li, B. W. Hsu, C. Y. Chen, C. I. Wu and H. W. Lin, ACS Appl. Mater. Interfaces, 2019, 50, 47054.

72 I. Tanaka, T. Kato, S. Ohkouchi and F. Osaka, J. Vac. Sci. Technol., A, 1990, 8, 567.

73 J. M. Gómez-Rodríguez, A. M. Baro, J. P. Silveira, M. Vazquez, Y. Gonzalez and F. Briones, Appl. Phys. Lett., 1990, 56, 36.

74 M. Kollár, L. Ćirić, J. H. Dil, A. Weber, S. Muff, H. M. Ronnow, B. Náfrádi, B. P. Monnier, J. S. Luterbacher, L. Forró and E. Horváth, Sci. Rep., 2017, 7,1 .

75 T. Chien, N. P. Guisinger and J. W. Freeland, J. Vac. Sci. Technol., B: Nanotechnol. Microelectron.: Mater., Process., Meas., Phenom., 2010, 28, C5A11.

76 A. Wang and T. Chien, Phys. Lett. A, 2018, 382, 739.

77 N. P. Guisinger, T. S. Santos, J. R. Guest, T. Y. Chien, A. Bhattacharya, J. W. Freeland and M. Bode, ACS Nano, 2009, 3, 4132.
78 F. Massee, S. de Jong, Y. Huang, J. Kaas, E. Van Heumen, J. B. Goedkoop and M. S. Golden, Phys. Rev. B: Condens. Matter Mater. Phys., 2009, 80, 140507.

79 Y. S. Hor, P. Roushan, H. Beidenkopf, J. Seo, D. Qu, J. G. Checkelsky, L. A. Wray, D. Hsieh, Y. Xia, S. Y. Xu and D. Qian, Phys. Rev. B: Condens. Matter Mater. Phys., 2010, 81, 195203.

80 M. C. Shih, B. C. Huang, C. C. Lin, S. S. Li, H. A. Chen, Y. P. Chiu and C. W. Chen, Nano Lett., 2013, 13, 2387.

81 L. F. Kourkoutis, J. Chakhalian, B. Gray, M. Kareev, N. P. Guisinger, D. A. Muller and J. W. Freeland, Nat. Commun., 2013, 4, 1.

82 T. Chien, J. Liu, A. J. Yost, J. Chakhalian, J. W. Freeland and N. P. Guisinger, Sci. Rep., 2016, 6, 19017.

83 E. T. Yu, Chem. Rev., 1997, 97, 1017.

84 B. Murali, S. Dey, A. L. Abdelhady, W. Peng, E. Alarousu, A. R. Kirmani, N. Cho, S. P. Sarmah, M. R. Parida, M. I. Saidaminov and A. A. Zhumekenov, ACS Energy Lett., 2016, 1, 1119.

85 S.-L. Lee, Y. Fang, G. Velpula, F.-P. Cometto, M. Lingenfelder, K. Müllen, K.-S. Mali and S. De Feyter, ACS Nano, 2015, 9, 11608.

86 S.-L. Lee, H.-J. Wu, Y.-J. Hsu, H.-H. Chen, H.-F. Hsu and C.-H. Chen, Chem. Commun., 2014, 50, 14093.

87 M. Kemerink, S. F. Alvarado, P. Müller, P. M. Koenraad, H. W. M. Salemink, J. H. Wolter and R. A. J. Janssen, Phys. Rev. B: Condens. Matter Mater. Phys., 2004, 70, 045202.

88 I. Caballero-Quintana, J. L. Maldonado, M. A. MenesesNava, O. Barbosa-García, J. Valenzuela-Benavides and A. Bousseksou, Adv. Electron. Mater., 2019, 5, 1800499.

89 B. Grévin, P. Rannou, R. Payerne, A. Pron and J. P. Travers, Adv. Mater., 2003, 15, 881.

90 Y. F. Liu, K. Krug and Y. L. Lee, Nanoscale, 2013, 5, 7936.

91 O. Amargós-Reyes, J. L. Maldonado, D. Romero-Borja, D. Barreiro-Argüelles, I. Caballero-Quintana, O. BarbosaGarcía and J. A. Gaspar, J. Mater. Sci., 2019, 54, 2427.

92 S.-L. Lee, Z. Yuan, L. Chen, K.-S. Mali, K. Müllen and S. De Feyter, J. Am. Chem. Soc., 2014, 136, 4117.

93 S.-L. Lee, J. Adisoejoso, Y. Fang, K. Tahara, Y. Tobe, K.-S. Mali and S. De Feyter, Nanoscale, 2015, 7, 5344.

94 L. K. Ono, E. J. Juarez-Perez and Y. Qi, ACS Appl. Mater. Interfaces, 2017, 9, 30197.

95 A. H. Slavney, R. W. Smaha, I. C. Smith, A. Jaffe, D. Umeyama and H. I. Karunadasa, Inorg. Chem., 2017, 56, $46 \mathrm{e} 55$.

96 F. Huang, M. Li, P. Siffalovic, G. Cao and J. Tian, From scalable solution fabrication of perovskite films towards commercialization of solar cells, Energy Environ. Sci., 2019, $12,518$.

97 J. Mohanraj, M. Stihl, E. Simon, O. Sicard, G. Schmidt, M. Fleischer, C. Neuber and M. Thelakkat, ACS Appl. Energy Mater., 2019, 2, 3469.

98 F. Matteocci, L. Cina, E. Lamanna, S. Cacovich, G. Divitini, P. A. Midgley, C. Ducati and A. Di Carlo, Nano Energy, 2016, 30, 162. 
99 L. K. Ono, N. G. Park, K. Zhu, W. Huang and Y. Qi, ACS Energy Lett., 2017, 2, 1749.

100 A. Kojima, K. Teshima, Y. Shirai and T. Miyasaka, J. Am. Chem. Soc., 2009, 131, 6050.

101 N. P. Guisinger, N. L. Yoder and M. C. Hersam, Proc. Natl. Acad. Sci. U. S. A., 2005, 102, 8838.

102 G. Franc and A. Gourdon, Phys. Chem. Chem. Phys., 2011, 13, 14283.

103 X. Che, B. Traore, C. Katan, M. Kepenekian and J. Even, Phys. Chem. Chem. Phys., 2018, 20, 9638.

104 S. Y. Leblebici, L. Leppert, Y. Li, S. E. Reyes-Lillo, S. Wickenburg, E. Wong, J. Lee, M. Melli, D. Ziegler, D. K. Angell and D. F. Ogletree, Nat. Energy, 2016, 1, 16093.

105 G. Niu, X. Guo and L. Wang, J. Mater. Chem. A, 2015, 3, 8970.

106 M. Shirayama, M. Kato, T. Miyadera, T. Sugita, T. Fujiseki, S. Hara, H. Kadowaki, D. Murata, M. Chikamatsu and H. Fujiwara, J. Appl. Phys., 2016, 119, 115501.

107 B. Philippe, B. W. Park, R. Lindblad, J. Oscarsson, S. Ahmadi, E. M. Johansson and H. Rensmo, Chem. Mater., 2015, 27, 1720.

108 K. Qin, B. Dong and S. Wang, J. Energy Chem., 2019, 33, 90.

109 A. R. Bowman, M. T. Klug, T. A. Doherty, M. D. Farrar, S. P. Senanayak, B. Wenger, G. Divitini, E. P. Booker, Z. Andaji-Garmaroudi, S. Macpherson and E. Ruggeri, ACS Energy Lett., 2019, 4, 2301.

110 K. H. Wang, L. C. Li, M. Shellaiah and K. W. Sun, Sci. Rep., 2017, 7, 1.

111 C. Wang, B. R. Ecker, H. Wei, J. Huang and Y. Gao, J. Mater. Chem. C, 2018, 122, 3513.

112 R. Comin, G. Walters, E. S. Thibau, O. Voznyy, Z. H. Lu and E. H. Sargent, J. Mater. Chem. C, 2015, 3, 8839.

113 A. M. A. Leguy, Y. Hu, M. Campoy-Quiles, M. I. Alonso, O. J. Weber, P. Azarhoosh, M. van Schilfgaarde, M. T. Weller, T. Bein, J. Nelson, et al., Chem. Mater., 2015, 27, 3397.

114 D. Ghosh, D. Acharya, L. Zhou, W. Nie, O. V. Prezhdo, S. Tretiak and A. J. Neukirch, J. Phys. Chem. Lett., 2019, 10, 5000 .

115 F. Zu, P. Amsalem, M. Ralaiarisoa, T. Schultz, R. Schlesinger and N. Koch, ACS Appl. Mater. Interfaces, 2017, 9, 41546.

116 J. Kim, S.-H. Lee, C.-H. Chung and K.-H. Hong, Phys. Chem. Chem. Phys., 2016, 18, 4423.

117 T. Gallet, D. Grabowski, T. Kirchartz and A. Redinger, Nanoscale, 2019, 11, 16828.

118 J. Endres, D. A. Egger, M. Kulbak, R. A. Kerner, L. Zhao, S. H. Silver, G. Hodes, B. P. Rand, D. Cahen, L. Kronik and A. Kahn, J. Phys. Chem. Lett., 2016, 7, 2722.

119 P. Schulz, E. Edri, S. Kirmayer, G. Hodes, D. Cahen and A. Kahn, Energy Environ. Sci., 2014, 7, 1377.

120 J. Bardeen, Phys. Rev. Lett., 1961, 6, 57.

121 C. Quarti, E. Mosconi, P. Umari and F. De Angelis, Inorg. Chem., 2017, 56, 74.
122 M. Zhang, H. Yu, M. Lyu, Q. Wang, J.-H. Yun and L. Wang, Chem. Commun., 2014, 50, 11727.

123 S. Luo and W. A. Daoud, Materials, 2016, 9, 123.

124 M. Ye, X. Hong, F. Zhang and X. Liu, J. Mater. Chem. A, 2016, 4, 6755.

125 J. Hieulle, X. Wang, C. Stecker, D. Y. Son, L. Qiu, R. Ohmann, L. K. Ono, A. Mugarza, Y. Yan and Y. Qi, J. Am. Chem. Soc., 2019, 141, 3515.

126 T. L. Cottrell, The Strengths of Chemical Bonds, Butterworth, London, 2nd edn, 1958.

127 J. J. Li, J. Y. Ma, Q. Q. Ge, J. S. Hu, D. Wang and L. J. Wan, ACS Appl. Mater. Interfaces, 2015, 7, 28518.

128 J. Hieulle, C. Stecker, R. Ohmann, L. K. Ono and Y. Qi, Small, 2018, 2, 1700295.

129 J. Li, B. Huang, E. N. Esfahani, L. Wei, J. Yao, J. Zhao and W. Chen, npj Quantum Mater., 2017, 2, 1.

130 M. Kratzer, O. P. Dimitriev, A. N. Fedoryak, N. M. Osipyonok, P. Balaz, M. Balaz, M. Tesinsky and C. Teichert, J. Appl. Phys., 2019, 125, 185305.

131 T. Supasai, N. Rujisamphan, K. Ullrich, A. Chemseddine and T. Dittrich, Appl. Phys. Lett., 2013, 103, 183906.

132 G. E. Eperon, T. Leijtens, K. A. Bush, R. Prasanna, T. Green, J. T. W. Wang, D. P. McMeekin, G. Volonakis, R. L. Milot, R. May and A. Palmstrom, Science, 2016, 354, 861.

133 M. Saliba, T. Matsui, K. Domanski, J. Y. Seo, A. Ummadisingu, S. M. Zakeeruddin, J. P. Correa-Baena, W. R. Tress, A. Abate, A. Hagfeldt and M. Grätzel, Science, 2016, 354, 206.

134 W. Xiang, Z. Wang, D. J. Kubicki, W. Tress, J. Luo, D. Prochowicz, S. Akin, L. Emsley, J. Zhou, G. Dietler and M. Grätzel, Joule, 2019, 3, 205.

135 L. Zhang, W. Cui, Z. Zang, F. Tian, X. Li and G. Qin, Sol. Energy, 2019, 188, 224.

136 T. Xiang, Y. Zhang, H. Wu, J. Li, L. Yang, K. Wang, J. Xia, Z. Deng, J. Xiao, W. Li and Z. Ku, Sol. Energy Mater. Sol. Cells, 2020, 206, 110317.

137 J. Duan, Y. Zhao, B. He and Q. Tang, Small, 2018, 14, 1704443.

138 G. Volonakis, A. A. Haghighirad, R. L. Milot, W. H. Sio, M. R. Filip, B. Wenger, M. B. Johnston, L. M. Herz, H. J. Snaith and F. Giustino, J. Phys. Chem. Lett., 2017, 8, 772.

139 B. A. Connor, L. Leppert, M. D. Smith, J. B. Neaton and H. I. Karunadasa, J. Am. Chem. Soc., 2018, 140, 5235 .

140 M. Chen, M. G. Ju, A. D. Carl, Y. Zong, R. L. Grimm, J. Gu, X. C. Zeng, Y. Zhou and N. P. Padture, Joule, 2018, 2, 558.

141 J. Kang and L. W. Wang, J. Phys. Chem. Lett., 2017, 8, 489.

142 J. Hieulle, S. Luo, D. Y. Son, A. Jamshaid, C. Stecker, Z. Liu, G. Na, D. Yang, R. Ohmann, L. K. Ono and L. Zhang, J. Phys. Chem. Lett., 2020, 11, 818.

143 M. A. Pena and J. L. G. Fierro, Chem. Rev., 2001, 101, 1981. 144 C. J. Won, Y. A. Park, K. D. Lee, H. Y. Ryu and N. Hur, J. Appl. Phys., 2011, 109, 084108. 
145 F. Zheng, Y. Xin, W. Huang, J. Zhang, X. Wang, M. Shen, W. Dong, L. Fang, Y. Bai, X. Shen and J. Hao, J. Mater. Chem. A, 2014, 2, 1363.

146 K. P. Marshall, M. Walker, R. I. Walton and R. A. Hatton, Nat. Energy, 2016, 1, 16178.

147 S. Y. Yang, L. W. Martin, S. J. Byrnes, T. E. Conry, S. R. Basu, D. Paran, L. Reichertz, J. Ihlefeld, C. Adamo, A. Melville and Y. H. Chu, Appl. Phys. Lett., 2009, 95, 062909.

148 Y. Wang, J. Tu, T. Li, C. Tao, X. Deng and Z. Li, J. Mater. Chem. A, 2019, 7, 7683.

149 Z. Zhao, J. Wu, F. Fang, T. Li, Y. Zhou and J. Wang, Mater. Res. Express, 2019, 6, 125534.

150 W. Ji, K. Yao and Y. C. Liang, Adv. Mater., 2010, 22, 1763.

151 Z. Fan, J. Xiao, K. Yao, K. Zeng and J. Wang, Appl. Phys. Lett., 2015, 106, 102902.

152 W. Huang, C. Harnagea, D. Benetti, M. Chaker, F. Rosei and R. Nechache, J. Mater. Chem. A, 2017, 5, 10355.

153 Z. Fan, K. Yao and J. Wang, Appl. Phys. Lett., 2014, 105, 162903.

154 F. Zheng, Y. Xin, W. Huang, J. Zhang, X. Wang, M. Shen, W. Dong, L. Fang, Y. Bai, X. Shen and J. Hao, J. Mater. Chem. A, 2014, 2, 1363.

155 S. Sharma, M. Tomar, A. Kumar, N. K. Puri and V. Gupta, J. Appl. Phys., 2015, 118, 074103.

156 J. F. Ihlefeld, N. J. Podraza, Z. K. Liu, R. C. Rai, X. Xu, T. Heeg, Y. B. Chen, J. Li, R. W. Collins, J. L. Musfeldt and X. Q. Pan, Appl. Phys. Lett., 2008, 92, 142908.

157 T. Ohsawa, K. Iwaya, Y. Okada, R. Shimizu, S. Shiraki and T. Hitosugi, in Oxide-based Materials and Devices VI: Int. Soc. Optics Phot, 2015, vol. 9364, p. 936407.

158 K. X. Jin, Y. F. Li, Z. L. Wang, H. Y. Peng, W. N. Lin, A. K. K. Kyaw, Y. L. Jin, K. J. Jin, X. W. Sun, C. Soci and T. Wu, AIP Adv., 2012, 2, 042131.
159 Z. Cai, Y. Kuru, J. W. Han, Y. Chen and B. Yildiz, J. Am. Chem. Soc., 2011, 133, 17696.

160 S. Chatterjee, A. Bera and A. J. Pal, ACS Appl. Mater. Interfaces, 2014, 6, 20479.

161 M. Ghiasi and A. Malekzadeh, Sep. Purif. Technol., 2014, $134,12$.

162 K. A. Stoerzinger, W. T. Hong, X. R. Wang, R. R. Rao, S. Bengaluru Subramanyam, C. Li, T. Venkatesan, Q. Liu, E. J. Crumlin, K. K. Varanasi and Y. Shao-Horn, Chem. Mater., 2017, 29, 9990.

163 N. Khellaf, A. Kahoul, F. Naamoune and N. Alonso-Vante, Electrocatalysis, 2017, 8, 450.

164 S. H. Hwang, S. Unithrattil, H. J. Lee, J. Song, H. J. Lee, G. Anoop, S. Lee and J. Y. Jo, Curr. Appl. Phys., 2019, 19, 950.

165 Y. Zhong, P. Chen, B. Yang, X. Zuo, L. Zhou, X. Yang and G. Li, Appl. Phys. Lett., 2015, 106, 263903.

166 J. S. Yun, A. Ho-Baillie, S. Huang, S. H. Woo, Y. Heo, J. Seidel, F. Huang, Y. B. Cheng and M. A. Green, J. Phys. Chem. Lett., 2015, 6, 875.

167 D. J. Wang, J. R. Sun, Y. W. Xie, Y. B. Li, L. G. Zhang, R. W. Wang and B. G. Shen, Appl. Phys. Lett., 2010, 97, 192503.

168 H. Ni, S. Da, K. Zhao, Y. C. Kong, H. K. Wong and S. Zhao, Appl. Phys. A, 2012, 108, 645.

169 Z. Q. Lu, H. Ni, K. Zhao, W. X. Leng, Y. C. Kong and H. K. Wong, Appl. Opt., 2011, 50, G23.

170 Q. Yang, X. Zuo, J. Yao, K. Zhang, H. Zhang, M. W. Khan, W. Wang, H. Tang, M. Wu, G. Li and S. Jin, J. Electroanal. Chem., 2019, 844, 34.

171 R. K. Vasudevan, A. Tselev, A. G. Gianfrancesco, A. P. Baddorf and S. V. Kalinin, Appl. Phys. Lett., 2015, 106, 143107. 